\title{
Engineering of superconductors and superconducting devices using artificial pinning sites
}

\author{
Roger Wördenweber \\ Peter Grünberg Institute (PGI), Forschungszentrum Jülich, D-52425 Jülich, Germany \\ Contact: r.woerdenweber@fz-juelich.de
}

\begin{abstract}
Vortex matter in superconducting films and devices is not only an interesting topic for basic research but plays a substantial role in the applications of superconductivity in general. We demonstrate, that in most electronic applications, magnetic flux penetrates the superconductor and affects the performance of superconducting devices. Therefore, vortex manipulation turns out to be a useful tool to avoid degradation of superconducting device properties. Moreover, it can also be used to analyze and understand novel and interesting physical properties and develop new concepts for superconductor applications. In this review, various concepts for vortex manipulation are sketched. For example, the use of micro- and nanopatterns (especially, antidots) for guiding and trapping of vortices in superconducting films and thin film devices is discussed and experimental evidence of their vortex guidance and vortex trapping by various arrangements of antidots is given. We demonstrate, that the vortex state of matter is very important in applications of superconductivity. A better understanding does not only lead to an improvement of the performance of superconductor components, such as reduced noise, better power handling capability, or improved reliability, it also promises deeper insight into the basic physics of vortices and vortex matter.
\end{abstract}

\section{Introduction}

With only a few exceptions, the electronic properties of type-II superconductors and the performance of superconducting devices are predominantly determined by the action of magnetic flux, i.e. the motion of magnetic flux lines (vortices). Magnetic flux penetrates the superconductor in form of fluxlines (vortices) that contain the smallest possible amount of magnetic flux, the magnetic flux quantum $\Phi_{\mathrm{o}}=\mathrm{h} / 2 \mathrm{e}=2.07 \cdot 10^{-15} \mathrm{~Wb}$. The motion of these vortices automatically leads to a local modification of the electric field, phase, and magnetic flux. Typical scenarios of this effect are illustrated in fig. 1:

(i) An applied current induces a Lorentz force $\vec{F}_{L}=\vec{J} \times \vec{\Phi}_{o}$ that acts on the vortices and is counteracted by the pinning force generated by defects in the material. Up to a critical current density $J_{c}$ the flux-line lattice is pinned and the superconducting current flows without dissipation. Above $J_{c}$ vortices start to move and generate an electric field (see fig. 1). Actually, flux creep and thermally assisted or activated flux-flow (TAFF) can lead to flux motion and thus electric fields already for $\mathrm{J}<\mathrm{J}_{\mathrm{c}}$. Nevertheless, for all applications that are based on the dissipation free DC current of the superconductor, the improvement of the pinning force is one of the major issues. In many cases it is even more important to improve $J_{c}$, than to enhance the superconducting transition temperature $T_{c}$ or the upper critical field $B_{c 2}$.

(ii) The motion of individual vortices typically leads to a degradation of the performance of superconducting electronic devices. This is illustrated in the second row of figures in fig. 1 for 
the case of a superconducting magnetometer, the SQUID. The motion of a single vortex leads to a telegraph-type noise in the sensor. The action of many vortices creates the typical $1 / \mathrm{f}$ noise in these sensors. Similar effects are known for other types of superconducting electronics. For instance in passive devices (.e.g. filters) or active devices (e.g. rapid single flux quantum logic, RSFQ) vortex motion leads to a reduction of the power-handling capability or enhances the bit error rate, respectively.

(iii) In contrast to the previous examples, the motion of the vortices can also be used for magnetic field or signal management (ranging from flux guides, flux focuser, ratchets, to filter) or data handling (RSFQ-type devices). This direction is generally called fluxonics.

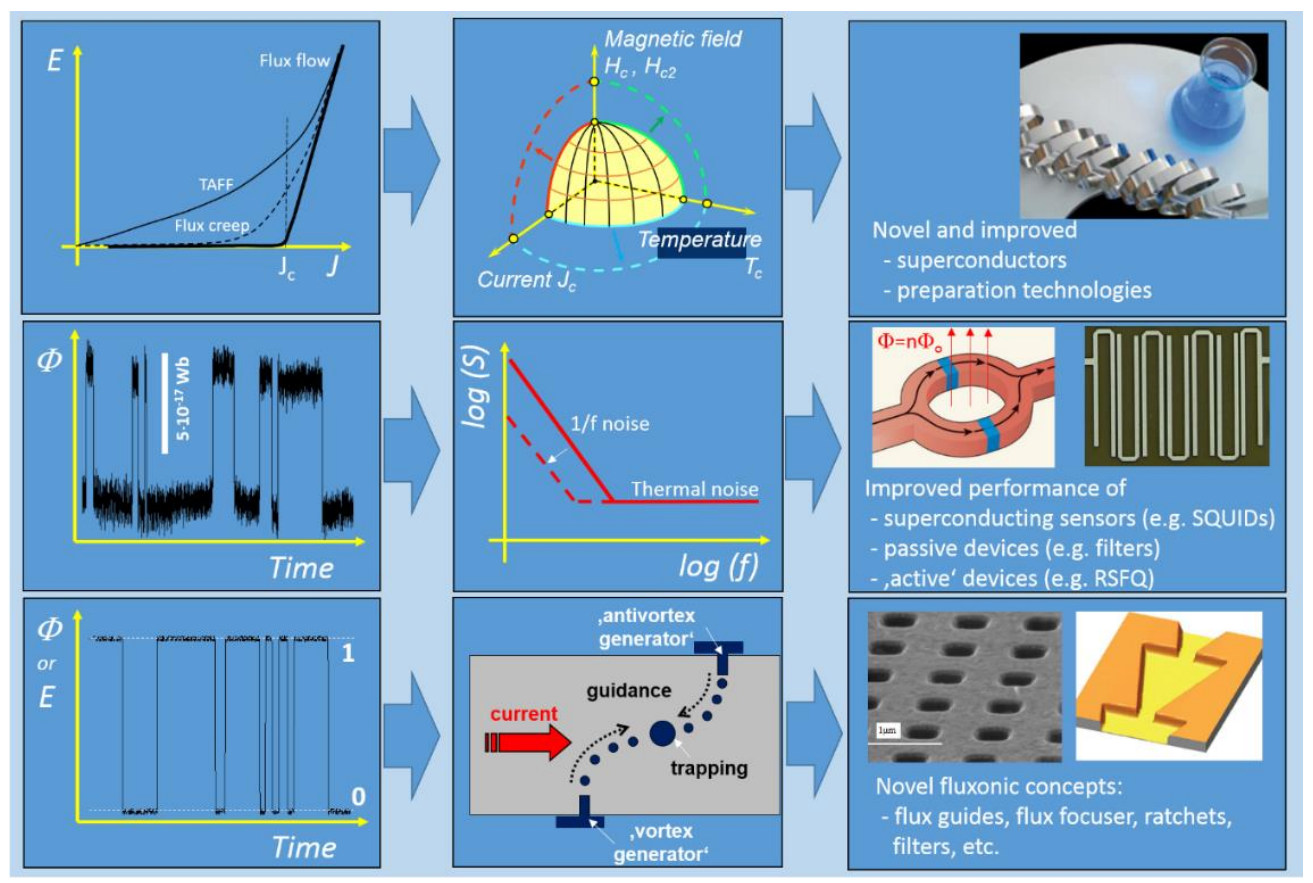

Fig. 1: Schematic sketches of the different scenarios of the impact of vortex motion on technological superconducting and superconducting devices. (i) (top row, from left to right) The nonlinear currentvoltage characteristic demonstrates the onset for flux flow at the critical current density $\mathrm{J}_{c}$, flux creep TAFF can lead to losses even below $\mathrm{J}_{\mathrm{c}}$. The resulting temperature-field-current phase-diagram defines the superconducting regime of loss-free current transport. In order to enhance the critical parameters (especially $J_{c}$ ) preparation technologies have to be optimized or new technologies have to be developed. (ii) (middle row, from left to right) Single vortex jumps lead to noise (telegraph noise), the motion of many vortices results in $1 / f$ noise that exceeds the noise floor (e.g. thermal noise) in superconducting devices. As a consequence the performance of the devices is reduced. (iii) (bottom row, from left to right) Vortex motion can also be used to switch between different states or generate a signal. This is the basic concept of flux motion based devices, the so-called fluxonic devices.

All three scenarios lead to one solution, the systematic manipulation of vortices which includes the pinning and/or the guided motion of vortices. This manipulation of vortices might be achieved by naturally grown defects. However it is evident that artificial defects present not only a crucial alternative to natural pinning sites. In quite a number of concepts they are absolutely necessary to achieve the desired goal (e.g. in case of fluxonic devices).

In the following sections, I will briefly introduce different types of artificial defects, basic aspects of their pinning interaction, the different possible types of flux motion between well separated pinning sites, and the basic demonstration of vortex-pin interaction and vortex guidance, before we move to aspects of artificial pinning sites in different applications like SQUIDs, passive devices, or fluxonics. 


\section{Artificial pinning sites}

There exist a large number of artificial pinning sites. In principle, any kind of modification of the superconducting material has an impact on the flux pinning potential of the superconductor. The question is whether the impact is large enough and suitable for the envisioned experiment or application. For the right choice of defect type the consideration of different possible classifications of artificial pinning sites is helpful:

- Pinning interaction and pinning strength: There exist two essentially different types of pinning interactions, which are the magnetic interaction and the core interaction. The magnetic interaction is essentially determined by the magnetic field gradient in the superconductor (characterized by the penetration length $\lambda$ ), whereas the core interaction arises from the interaction of locally distorted superconducting properties with the variation of the superconducting order parameter (characterized by the coherence length $\xi$ ). Furthermore, trapping of quantized flux $n \Phi_{\circ}$ can be achieved by extended voids or holes. There exist predictions for the individual pinning strength for a number of defect types.

- Size: The typical size of a pinning site based on core interaction (e.g. $\delta \kappa$ or $\delta T_{c}$ pinning, the alternative magnetic interaction is not considered here) should be comparable to the size of the normal core of the vortex which is equivalent to the coherence length $\xi$. Since the coherence length of most superconductors of interest is of the order of 1-4nm (exceptions are $\mathrm{Nb}$ and $\mathrm{Pb}$ with $40 \mathrm{~nm}$ and $51-83 \mathrm{~nm}$, respectively, see Tab. 1 of the Tutorial) the pinning sites have to be extremely small. Nevertheless, larger defects can also affect flux lines. In this case, pinning can be provided for instance by the boundary of the defect or the mechanical strain field generated by the defect. Alternatively, extended defects can trap quantized flux $\Phi=n \Phi$ 。 in the form of individual flux quanta $\Phi_{\text {o }}$ (e.g. for partially etched holes, so-called blind holes) or multi-quanta vortices with different vorticity $\mathrm{n}$ (e.g. for holes, so-called antidots). The use of antidots is of special interest for guidance and trapping of flux and will be the major type of artificial defect to be considered throughout this review.

- Orientation and dimension: The 'classical' pinning site is a point defect, which can be considered to be a OD pinning sites. 1D pinning sites can be generated for instance by irradiation, whereas typical 2D pinning sites are represented by all types of surfaces including the surfaces of the sample itself, surfaces of extended defects, interfaces, and even modulations of the superconductivity in highly anisotropic superconductors. The latter leads to the so-called intrinsic pinning for instance observed in ceramic high- $T_{c}$ materials. Extended $3 D$ defects should rather be considered to trap flux (see above). Evidently, for the 1D and 2D pinning sites the orientation of the defect plays an important role. The pinning force of the defect is largest for a perfect alignment of flux and defect (e.g. magnetic lock-in effect).

- Connectivity: Connected superconducting areas with locally tailored electrical properties (mainly 'channels for vortex motion') are used mainly for vortex guidance. Non-simply connected micro or nanostructures will caused a long-range electronic or magnetic interaction between vortex and artificial structure. Generally, the flux pinning and flux transport is different for both arrangements.

Preparation: There exist a large variety of preparation methods for artificial defects. During the formation of the superconducting material, deviations from the stoichiometry, doping, or addition of other phases or nanoparticles automatically create defects which are usually statistically distributed. These in-situ methods are used, for instance, for the improvement of the critical current density in large-scale applications like the fabrication of superconducting cables and coated conductors. However, after preparation of the material, defects can also be introduced by many ex-situ techniques. Irradiation with energetic particles (ions, protons, $\alpha$-particles, or even electrons) will introduce defects 
in the material. The nature of the defect depends on the type of irradiation, the particle energy and dose, and the superconducting material itself. For instance, heavy-ion irradiation can produce columnar tracks or implantation of the ion depending on the energy of the ion. Irradiation techniques ([1], and references cited within) can be used to generate statistically distributed pinning sites or patterned pinning structures if masks or focused beam lines are used. However, the best option to position and arrange artificial defects is given by patterning technologies. Via lithography and etching, nano or micro-structures can be generated in the superconducting layer, the substrate (carrier of the layer) before deposition, or in additional layers (e.g. magnetic films) that couple to the superconductor. These structures will affect the pinning and motion of vortices in the superconductor.

- Reconfigurability: Typically, pinning sites are not reconfigurable. This also holds for artificial defects. Nevertheless there are attempts to produce reconfigurable defects for instance using soft-magnetic dots (with switchable magnetization), ferroelectric top layers (with reconfigurable polarization), or locally reconfigurable stoichiometry (e.g. local modification of the oxygen content in ceramic high- $\mathrm{T}_{\mathrm{c}}$ superconductors).

From this list, it is obvious that one can and should choose the respective type of defect according to the needs of the experiment or the application. Nevertheless, a number of particularly interesting patterns and defect types are briefly described below.
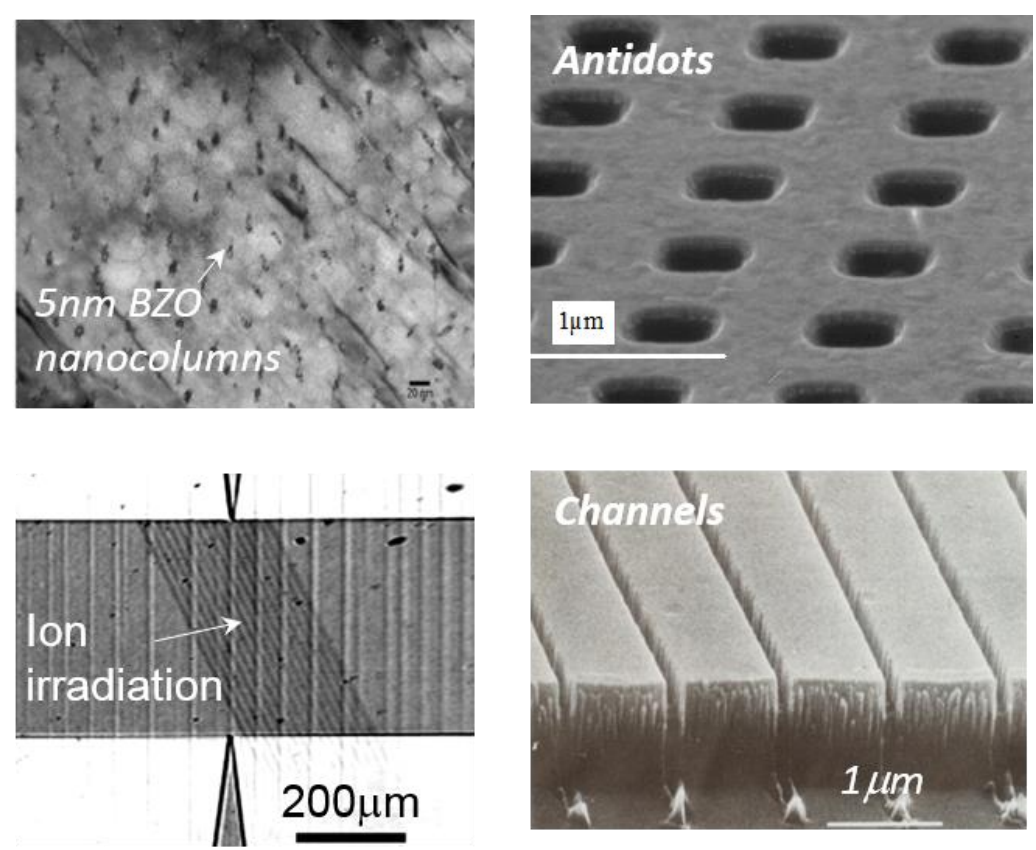

Fig. 2: SEM images of artificial defects ranging from $\mathrm{BaZrO}_{3}$ nanoparticles, antidots, or heavy-ion patterned channels in $\mathrm{YBa}_{2} \mathrm{Cu}_{3} \mathrm{O}_{7-\delta}$ thin films [4-8] to patterned channels in NbN/NbGe bilayer [2,3,9].

Artificial channels: The most obvious concept to provide a controlled vortex motion (the so-called guided vortex motion) is given by the pattering of narrow channels into superconducting material. This can be done by etching channel structures into a single layer leading to modification of the pinning force due to thickness variation, local modification of the superconducting properties (for instance via heavy ion lithography or antidots), or by combining two layers of superconducting material with different pinning properties.

Combination of weak and strong-pinning material: An intriguing example of easy vortex flow channels consists of a weak-pinning bottom layer (e.g. amorphous $\mathrm{NbGe}$ ) combined with a strong-pinning top layer (e.g. $\mathrm{NbN}$ ), into which the small channels are etched (see fig.2) $[2,3]$. Since the pinning force in 
the channel is extremely weak, the vortices in the channels predominantly experience the vortexvortex interaction with the row of the strongly pinned vortices at the edge of the channel within the $\mathrm{NbN}$. The commensurability between the vortex lattice with a field dependent lattice parameter $a_{0}$ $=b_{o}\left(\phi_{o} / B\right)^{1 / 2}$ and the channel size leads to periodic oscillations of the volume pinning force. Vortices move within the channels if the total driving force on the vortices in the channel exceeds the shear forces at the channel edges.

However, rather more complex channel patterns can be used. An example of a ratchet system using the same system (week-pinning a-NbGe in combination with a strong-pinning and patterned $\mathrm{NbN}$ toplayer) is shown in fig. 1 [10]. Using an asymmetric, 'fyke-like' pattern, the vortex motion is not only guided, it can be rectified and, thus, create a fluxonic ratchet.

Similar structures are possible for high- $\mathrm{T}_{\mathrm{c}}$ films. However, for these materials it turns out to be better to locally modify the superconducting properties and create channels in high- $\mathrm{T}_{\mathrm{c}}$ films, for instance by using heavy ion lithography or antidots.

Heavy Ion Lithography: Ion irradiation of HTS films offers a unique possibility to create a wide range of different defects and to tailor the electrical and superconducting properties [1]. Depending on the species of ions used during the irradiation, their energy and fluence, nanoscale columnar pinning centres can be created that locally enhance or diminish the pinning properties. Whereas the irradiation with relatively low fluence of high energy heavy ions leads to an enhancement of the critical current due to the strong vortex pinning at columnar defects, relatively high fluence leads to a reduction of the critical properties. Thus, the superconducting properties can be controlled and modulated locally. The preparation of artificial channels for flux motion has been demonstrated for this technology [7].

Dots and Antidots: In contrast to simply connected structures, dots and holes (antidots) offer a number of advantages for the manipulation of vortices: (i) dots or antidots of various size, properties and shapes can be used or even combined, (ii) they can be positioned more or less at wish, even quite complex arrangements are possible, (iii) they can be used as pinning (single-flux quanta) or trapping (multi-flux quanta) sites as well as for the guidance of flux, and (iv) the motion of flux between dots or antidots can be examined and used.

In contrast to other pinning defects, which have to be of the order of the size of the superconducting coherence length $\xi$, antidots with sizes much larger than $\xi$ will trap magnetic flux very effectively [4]. The advances in lithography techniques and the possible use of antidots in applications (e.g., SQUIDs [11,12], vortex diodes [13-15], microwave devices [16-18]) have led to a renewed interest in the research of superconducting films containing antidots or antidot lattices. Antidots have been successfully prepared in films of conventional superconductors (typically weak pinning $\mathrm{Pb}, \mathrm{V}$, or a-WGe thin films, $\mathrm{Pb} / \mathrm{Ge}$ multilayers, $\mathrm{Pb} / \mathrm{Cu}$ bilayers or $\mathrm{Nb}$ foils) [19-29] as well as HTS material (YBCO) [4$6,11,12,30]$. Intensive studies have been performed of

(i) commensurability effects (matching effects) between the antidot lattice and vortex lattice,

(ii) multiquanta formation in the antidots,

(iii) guided motion along rows of antidots,

(iv) visualization of vortex motion, and

(v) implementation of antidots in cryoelectronic devices.

Due to their unique properties, I will concentrate in this chapter on the role of antidots and their options and prospects for the manipulation of vortices. Nevertheless, most concepts can easily be used for the understanding of other types of artificial defects and their use in devices. 


\section{Vortex Manipulation via Antidots}

In this section, basic aspects of vortex manipulation via antidots are given, ranging from the vortexantidot interaction, multi-quanta vortex formation, commensurability or matching effects, different modes of vortex motion between antidots, to vortex guidance.

\subsection{Vortex-Antidot Interaction and Multi-Quanta Vortices}

In contrast to the classical pinning defects, antidots represent extended ( $>\xi)$ and 'hollow' defects. They act more like a container for quantized flux $\Phi=n \Phi_{0}$. Moreover, although obviously $T_{c}$ is zero inside the antidot, the vortex-antidot interaction differs from the classical $\delta T_{c}$ interaction.

The interaction energy between a vortex and a small insulating cylindrical cavity (analogue to an antidot) has been calculated using the London approximation [14] and an alternative approach using the analogy between a vortex close to an antidot and a charge line in an infinite dielectric close to a cylindrical cavity of different dielectric permittivity [15]. Later, the calculations have been extended to arbitrarily large cavities [31]. It is demonstrated that the interaction energy is identical to the one between a vortex and the straight edge of a superconductor (Bean-Livingston barrier [32]) when the radius of the antidot goes to infinity. Although the precise form of the interaction potential between a vortex and a cylindrical antidot is slightly different in these studies [14,15,31], the main conclusions are identical.

Based upon a series expansion of Bessel functions $K_{o}$ of the second kind, the free energy of a vortex at a radial distance $r$ from an antidot with radius $r_{o}$ is given by $[14,15]$

$$
F(r)=\frac{\Phi_{o}^{2}}{4 \pi \mu_{o} \lambda^{2}}\left[K_{o}\left(\frac{\xi}{\lambda}\right)+n^{2} K_{o}\left(\frac{r_{o}}{\lambda}\right)+2 n K_{o}\left(\frac{r}{\lambda}\right)+\ln \left(1-\frac{r_{o}^{2}}{r^{2}}\right)\right],
$$

for $\xi<\left(r, r_{0}\right)<<\lambda$ and $n$ representing the number of flux quanta that are already trapped in the antidot. The elementary vortex-antidot interaction is then given by the derivative, $f_{V A}=-\partial F / \partial$.

The vortex-antidot interaction depends upon the magnetic flux $\Phi=n \Phi_{o}$ trapped in the antidot (see fig. 3). The interaction between a vortex and an 'empty' antidot is attractive for all vortex-antidot distances. As soon as one flux quantum is trapped in the antidot, the interaction potential changes and a surface barrier emerges at the edge of the antidot. The height of the barrier increases with increasing number of trapped flux quanta. This automatically defines a saturation number $n_{s}$. For $n \geq n_{s}$ the interaction becomes repulsive, no additional flux can be trapped by the antidot. The saturation number depends upon the size of the antidot. For small antidots (single antidot with $r_{0} \ll \lambda$ ) the saturation number can be approximated by [14]

$$
n_{s} \cong \frac{r_{o}}{2 \xi(T)}
$$

the pinning force per unit length depends on the radial distance between vortex and antidot and has a maximum value close to the antidot edge

$$
f_{p, \max } \approx \frac{\Phi_{0}^{2}}{4 \sqrt{2} \pi \mu_{o} \lambda^{2} \xi}\left(1-\frac{n}{n_{s}}\right),
$$

i.e., the maximum vortex-antidot interaction decreases with increasing occupation number and becomes zero for $n=n_{\text {s. }}$.

Generally, the saturation number depends upon temperature, i.e. it increases with decreasing temperature. Furthermore, a consequence of a saturation number $n_{s}>1$ is the formation of so called 
multi-quanta vortices. An experimental proof of the existence of multi-quanta vortices is given in fig. 3. It shows the magneto-optic image of an YBCO film with antidots with a diameter of $2 \mu \mathrm{m}$ exposed to a $1 T$ external field. After removal of the external field the antidots trap a field of approx. $280 \Phi_{\text {o }}$ which agrees with the prediction of the theory (eq. (2)).
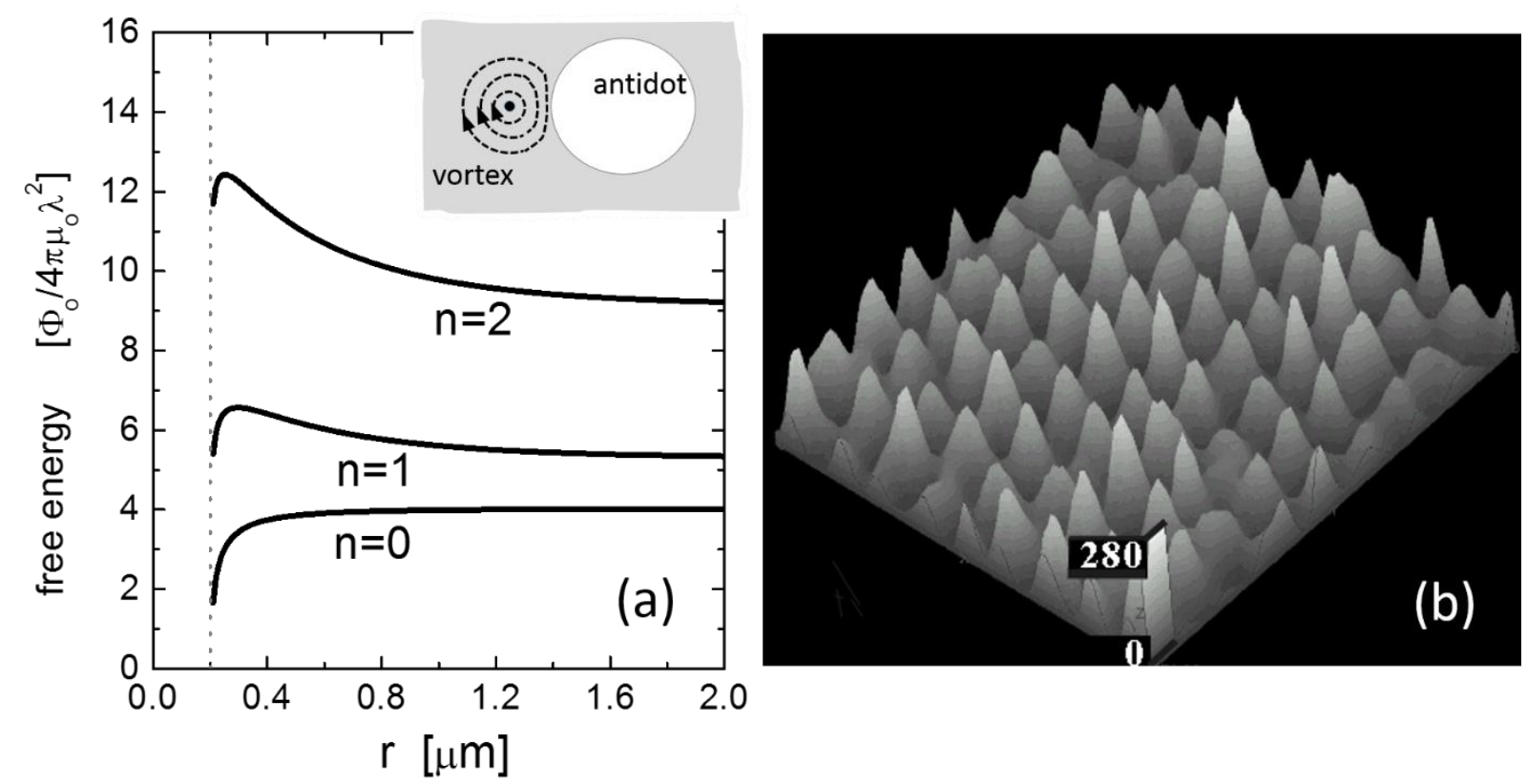

Fig. 3: Free energy of a vortex as function of the radial distance from an antidot for different occupation numbers $n$ of the antidot $\left(\lambda=600 \mathrm{~nm}, \kappa=50\right.$, and $r_{o}=200 \mathrm{~nm}$ ), (inset) sketch of a vortex interacting with a cylindrical cavity formed by an antidot with radius $r_{0}$, and (b) reconstruction of the trapped magnetic flux distribution obtained from a magneto-optic image that was recorded at $7 \mathrm{~K}$ in zero-field after applying at field of $1 \mathrm{~T}$ [33]. The local field shows clear maxima at the position of the antidots (triangular lattice, $d_{A A}=10 \mu \mathrm{m}, r_{0} \approx 1 \mu \mathrm{m}$ ). The scale for the height of the peaks is given in numbers of trapped flux quanta.

\subsection{Matching or commensurability effect}

The matching or commensurability effect represents an ideal tool to demonstrate the interaction between a regular (typically hexagonal) vortex lattice and a regular defect lattice. Furthermore it provides a first approach to improve the flux pinning for discrete magnetic fields (matching fields).

Since the vortex lattice parameter $a_{0}$ depends on the applied field $\left(a_{o}=1.15\left(\Phi_{o} / B\right)^{1 / 2}\right.$ or $a_{o}=\left(\Phi_{o} / B\right)^{1 / 2}$, for a hexagonal or square lattice, respectively) it can be varied and, thus, 'matched' for discrete magnetic fields to a regular lattice of defects. For these so-called matching fields, maxima in the flux pinning occur. Matching effects are typically demonstrated via measurements of the critical current, resistance, or ac susceptibility of a film structure patterned with a periodic array of artificial defects, e.g. square and triangular arrays of sub- $\mu \mathrm{m}$ antidots $[4,6,19,27,34]$.

While periodic pinning arrays show these enhanced pinning properties for a few discrete field values, at which the vortex density and defect density are integer multiples of each other, quasiperiodic arrays represent a valuable alternative, as they have many more built-in periodicities to which the vortex lattice can be approximately commensurate [35-37]. This leads to broadened maxima of the pinning efficiency and to a reduced sensitivity of the sample properties to magnetic field variations. Therefore quasiperiodic arrays of artificial defects represent a first option to improve flux pinning over a larger field range. 
Matching effects can also be demonstrated via noise measurements. Since this automatically leads us to an important aspect of the use of strategically positioned artificial defects (see section 4.2) an example of this demonstration is briefly described in the following.

In this experiment [38] a periodic array of artificial defects (YBCO film with a square lattice of antidots) is mounted in flip-chip configuration on a YBCO bicrystal rf-SQUID (see sketch in fig. 4a). Typical lowfrequency noise-spectra of this arrangement are given in fig. 4a. As expected, the lowest flux noise is recorded for zero magnetic field. At non-zero field, the low frequency noise strongly depends upon the exact value of the applied magnetic field. In contrast to the standard field dependence $S_{\Phi} \propto B$, it varies non-monotonically and over several orders of magnitude in noise level. For example, the noise at fields of $750 \mathrm{nT}$ or $900 \mathrm{nT}$ is more than 2 orders of magnitude larger than for the matching fields $M_{1}$ and $M_{2}$ (828nT and $845 \mathrm{nT}$, respectively) at which the noise level of the zero-field spectrum is recorded. The matching fields and matching configurations are illustrated in fig. 4b. Actually, although the array of antidots can reduce the noise for the matching fields, it can also enhance the noise for non-matching conditions.

Later we will see that in many cases a 'strategic' arrangement of artificial defects is necessary to optimize the benefit from artificial defects in superconducting devices.
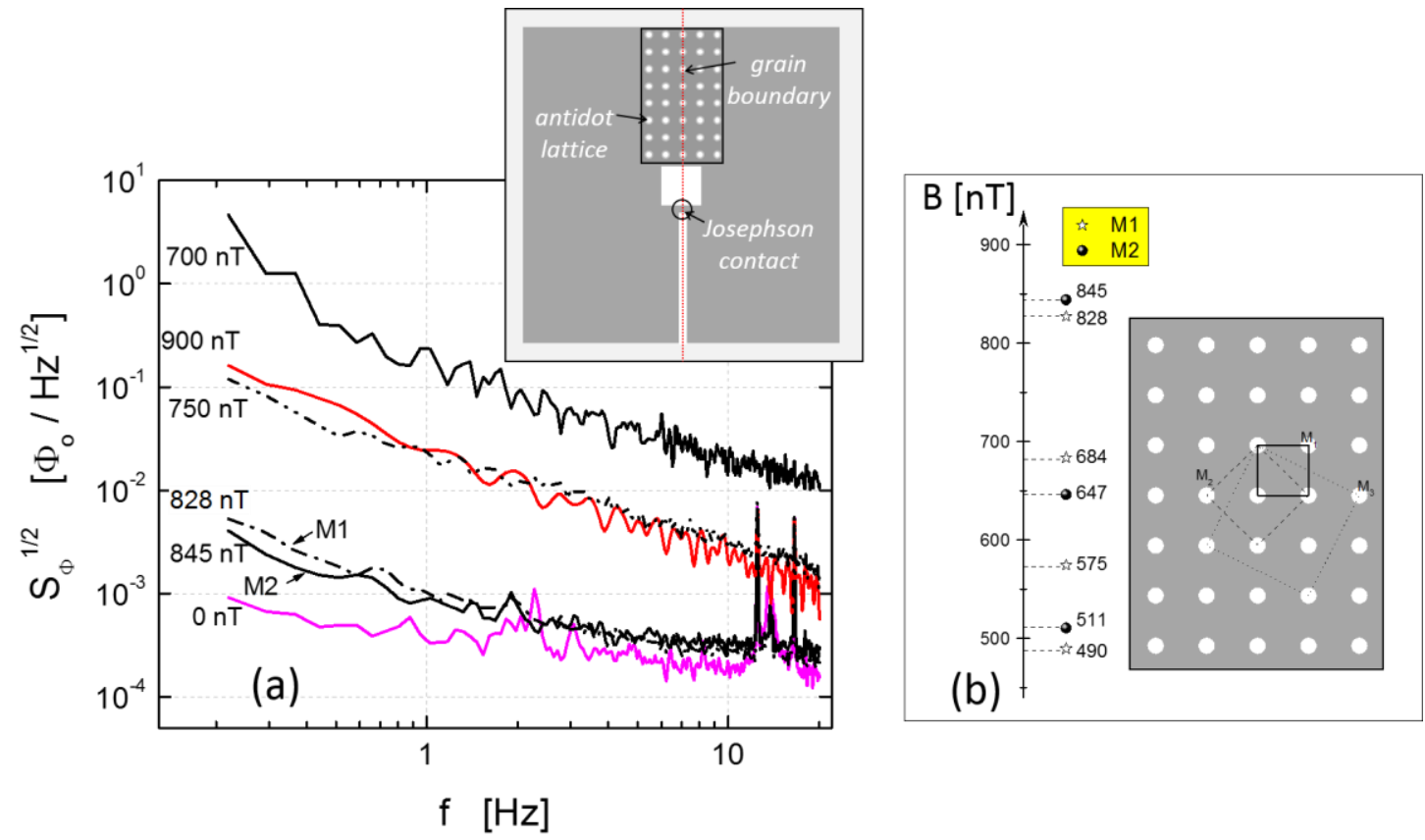

Fig. 4: (a) Low-frequency noise spectra of a bicrystal rf-SQUID (sketch) with a square antidot lattice on top of the grain boundary of the washer for different magnetic fields [38]. $M_{1}$ and $M_{2}$ label two matching conditions (see sketch fig. (b)). The inset (a) shows a sketch of the experimental arrangement. A YBCO film with square antidot lattice $\left(d_{A A}=5 \mu \mathrm{m}\right)$ is mounted in flip-chip configuration on top of the grain boundary of a bicrystal rf-SQUID. One of the main axes of the antidot lattice is oriented parallel to the grain boundary. The matching fields $M_{i}(i=1,2)$ in the field range 450 to $900 n T$ are given in (b). They are calculated for different vortex configurations (see schematic sketch for $\mathrm{i}=1-3 \mathrm{in}$ (b)) that match the square antidot array with a periodicity of $5 \mu \mathrm{m}$. For details, especially about the different matching conditions, see ref. [38].

\subsection{Guided vortex motion}

Probably even more important than flux trapping is the guidance of vortices by artificial defects. Guided motion has been demonstrated in conventional weak pinning superconductors $[2,3,9]$ and HTS 
thin films [7] using channels prepared via patterning or heavy ion irradiation (see also fig. 2). However, an intriguing and more flexible method of vortex guidance is provided by special arrangements of antidots, i.e. rows of antidots [33,39].

A first basic explanation of the angular dependence of the guidance of vortices via rows of antidots is given by the n-channel model [6]. The sketch in fig. 5 illustrates the angular dependence of the Hall voltage $V_{\text {Hall }}$ predicted in a simplified 1-channel model, in which the flux is expected to drift only along rows of antidots. The orientation of the rows is given by the angle $\gamma$. The component of the Lorentz force, which compels vortices to move along the antidot rows (i.e. guided motion), is $F_{\text {guid }}=F_{L} \cos \gamma$, where $F_{\mathrm{L}}$ is the modulus of the Lorentz force: $F_{\mathrm{L}}=\left|F_{L}\right|$. The components parallel and perpendicular to the applied current are

$$
\begin{aligned}
& F_{x, \text { guid }}=F_{\text {guid }} \cdot \sin \gamma=F_{L} \cdot \cos \gamma \cdot \sin \gamma \\
& F_{y, \text { guid }}=F_{\text {guid }} \cdot \cos \gamma=F_{L} \cdot \cos ^{2} \gamma
\end{aligned}
$$

with $F_{x, \text { guid }}$ and $F_{y, \text { guid }}$ contributing to the Hall and longitudinal voltage signal, respectively. The experimentally determined angular dependence of the Hall voltage roughly obeys the simple relation $V_{\text {Hall }} \propto F_{\mathrm{L}} \cos \gamma \sin \gamma$ obtained in this '1- channel model' [6]. Actually, it has to be considered, that vortices can also move with some probability between antidots of neighboring rows. These additional channels of vortex motion become important for large angles $\gamma[40]$ leading to a more general expression

$$
V_{\mathrm{H}} \propto \sum_{i} P_{i}(\gamma) F_{\mathrm{L}} \cos \gamma \sin \gamma,
$$

where the summation is performed over all possible channels of vortex motion, and $P_{i}(\gamma)$ is the angledependent probability of the motion along the $i$-th channel.

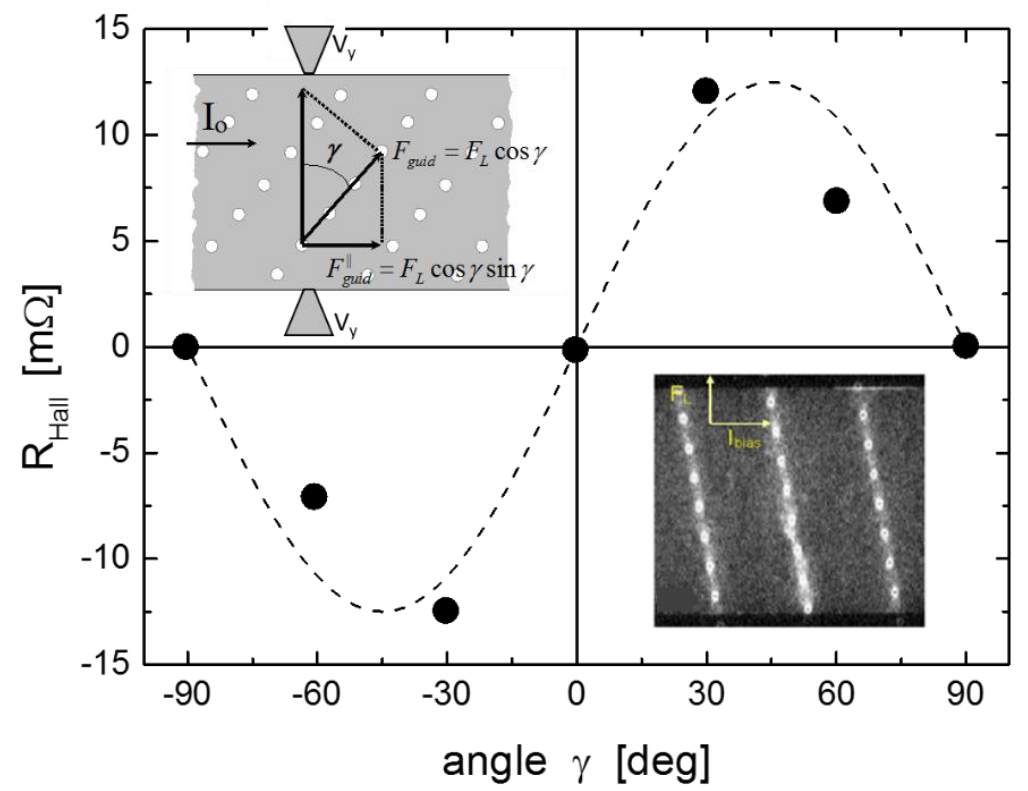

Fig. 5: Angular dependence of the Hall resistance (solid symbols) and 1-channel approximation (dashed line). The sketch illustrates the angular dependence of the Hall signal according to the $n$-channel model [6], the image shows an experimental demonstration of the guidance of vortices for rows of antidotes arranged at an angle $\gamma=-9^{\circ}$ with respect to the Lorentz force obtained via laser scanning microscopy [40]. 
In order to obtain a more detailed insight into the guidance of vortices via rows of antidots, resistive 6-probe dc measurements can be performed on superconducting films equipped with rows of antidots that simultaneously record the longitudinal voltage signal and the Hall signal $[6,30]$. Fig. 6 shows schematically the typical sample design that is suitable for simultaneous recording of the longitudinal voltage and the Hall voltage.

The longitudinal voltage (contacts $V_{x}$ ) represents the standard parameter to characterize the critical properties of a superconducting stripline. In the normal regime, it represents the normal state resistivity, whereas in superconducting regime it is generated by the component of vortex motion along the Lorentz force. In the latter case, the longitudinal voltage is a measure of the flux transfer across the stripline that is collected between the longitudinal voltage contacts labelled $V_{x}$ (integral signal). It characterizes the average velocity component $\left\langle v_{y}\right\rangle$ of vortices in the stripline. In contrast, in the superconducting regime the Hall signal characterizes the complementary velocity component $\left\langle v_{x}\right\rangle$ of vortices. It represents a more local analysis of the vortex motion that is restricted to the vicinity of the contact pair (Hall contacts $V_{y}$ in fig. 6).

High- $T_{c}$ materials typically reveal the, so-called, anomalous Hall effect (AHE), i.e. a sign inversion of the Hall signal below $T_{\mathrm{c}}$ [41-50]. A typical example is given in fig. 6a. The AHE is caused by vortex motion. Close to $T_{\mathrm{c}}$ when pinning is very weak, the impact of the Magnus force on the moving vortex leads to an additional component of the vortex motion perpendicular to the Lorentz force (i.e. parallel to the current) [50]. The resulting AHE competes with the guidance of vortices by rows of antidots (guided motion (GM)). Depending on the orientation of the rows of antidots, the AHE is suppressed or enhanced. This is sketched and demonstrated for different experiments in figs. $6 b-d$.
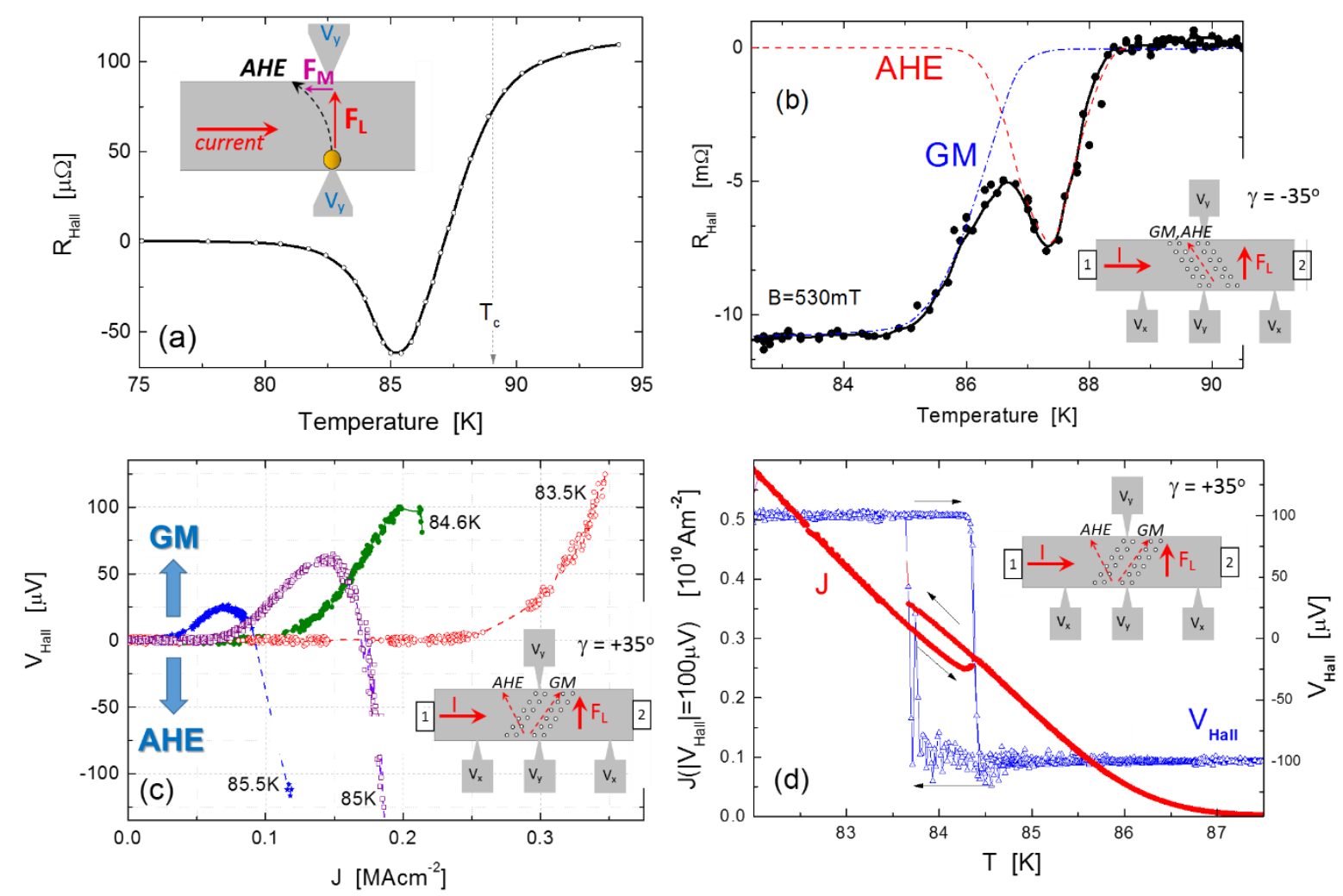

Fig. 6: Demonstration of guided vortex motion via Hall measurements. (a) The Hall resistance for an unpatterned YBCO film shows the typical anomalous Hall effect (AHE), i.e., the sign-inversion at temperatures just below $T_{c}$, (b) due to the tilted rows of antidots the guided motion (GM) enhances the AHE and extends the regime of negative Hall resistance to low temperatures [6], (c) in case of a tilt 
angle opposing the direction of the AHE a sign-inversion is visible for the current dependence of the Hall voltage for temperatures close to $T_{c}$, i.e. the direction of collective flux flow changes from $G M$ (small current) to AHE (large current), (d) another demonstration of the change of the direction from AHE to GM is given by the longitudinal current which generates a constant flux flow perpendicular to the current (i.e. a constant absolute Hall voltage, here $\left|\mathrm{V}_{\text {Hall }}\right|=100 \mu \mathrm{V}$ ). A detailed description of the experimental details is given in the text, the sketches in the figures illustrate the arrangement of the experiment $\left(V_{x}\right.$ and $V_{y}$ mark the longitudinal and Hall contacts, respectively, 1 and 2 are the current contacts), the orientation of the rows of antidots $\gamma$, and forces [30].

If the directions of the Magnus force and the GM coincide $\left(\gamma<0^{\circ}\right)$ a negative Hall signal is expected for both effects. This is demonstrated for YBCO films equipped with rows of antidots that are tilted with an angle $\gamma=-35^{\circ}$ (fig. 6b) [6]. However we can clearly distinguish the impact of the AHE and the GM. At $T_{c}$ superconductivity sets in and vortices are formed. However, vortex pining is small compared to the thermal activation of the vortices, the superconductor is in the reversible regime. As a consequence there is no guidance of the vortices. Thus, the vortices will follow the Lorenz force and Magnus force and show the typical AHE. If the temperature is reduced further, pinning sets in. The 'background' pinning due to defects in the superconductor will reduce the effect of the Magnus force, i.e. the AHE will vanish (see also fig. 6a). However, at the same time the pinning interaction of the antidots starts to guide the vortices. As a result a second decrease of the Hall resistance with decreasing temperature is visible (fig. 6.b).

If the directions of the Magnus force and the GM are opposite $\left(\gamma>0^{\circ}\right)$ we can even observe switching effects, i.e. the collective motion of vortices switches from the AHE to the GM direction. This is shown for two experiments in figs. $6 \mathrm{c}$ and $\mathrm{d}$. In the first case (fig. $6 \mathrm{c}$ ) current -voltage characteristics are recorded at temperatures close to $T_{c}$. However, instead of the longitudinal voltage, the Hall voltage is recorded. An inversion of the sign indicates the inversion of the direction of the motion of the vortices from antiparallel to parallel to the applied current. Above the critical current the voltage increases (GM). If the current is increased further, the guidance vanishes and the Hall signal becomes negative (AHE).

In the last example (fig. 6d) we again observe the switching from the AHE at high temperature to the GM at low temperature. In this experiment, the Hall voltage (absolute value) has been kept constant $\left(\left|V_{\text {Hall }}\right|=0.1 \mathrm{mV}\right)$. The explanation of the switching, which is visible in the hysteretic change from a negative to a positive Hall voltage as well as in the hysteretic change of the applied current, is similar to the one given for the experiment shown in fig. $6 \mathrm{~b}$ above. For more details see ref. [30].

Generally, guidance of vortices via antidot arrangements is now well established. However, it depends in a complicated way on the relation (amplitude and direction) of the different forces and potentials acting on the vortices. The major forces to be considered are the 'background' pinning force of the superconductor, the vortex-antidot interaction, driving forces (e.g., Lorentz force), Magnus force, and thermal activation. Some of these interactions depend on temperature, others on their orientation direction. This can lead to switching of the direction of vortex motion as function of temperature (see fig. 6.d), current density (see fig. 6.c), or orientation of antidot arrangement (see fig. 6). As a consequence, guidance of vortices strongly depends upon temperature, driving force, and geometrical arrangement of the antidots. If additionally anisotropic pinning potentials are introduced (e.g. via asymmetrically shaped antidots) preferentially directed vortex motion can be induced leading to more complex concepts of for instance ratchets, vortex diodes, or vortex filters. 


\subsection{Vortices at high velocity}

Studies of vortex matter are predominantly focused on the limit of small driving forces and small frequencies, i.e. the onset of vortex dynamics in the limit of small vortex velocities. However, a number of superconductor applications like coated conductors, fault-current limiters or microwave devices operate at high power levels or high frequencies and, therefore, potentially in the regime of high vortex velocities. This scenario also applies for the guided vortex motion in a superconductor equipped with an array of antidots if flux is expected to shuttle between closely spaced antidots at high frequencies. For example in case of an antidot spacing of $1 \mu \mathrm{m}$ a 'classical' Abrikosov vortex would have to travel with a velocity of $2 \mathrm{~km} / \mathrm{s}$ in order to oscillate between both antidots at a frequency of $1 \mathrm{GHz}$. In these cases, nonequilibrium effects are expected to occur in the superconductor.

In principle three different approaches to this scenario are feasible:

(i) With increasing vortex velocity the classical Abrikosov vortices gradually start to deform. Nevertheless, they can be treated as 'ballistic vortices' with slightly modified properties.

(ii) At a characteristic velocity, the so-called critical velocity, the vortices abruptly change their properties [51]. This high-velocity range is often associated with a different vortex state, the so-called kinematic vortices.

(iii) Finally, similar to the vortex motion in nano- or microbridges, the flux transport could take place in form of phase slip [52,53]. As a consequence flux transport at much higher velocities and, thus, frequencies would be possible. Moreover, a Josephson-type behavior should be visible.

Pulsed measurements of the current-voltage characteristics (IVC) on classical superconductors [54,55] as well as HTS material [56-58] demonstrate, that scenario (i) does not apply. At a critical voltage, the IVC changes abruptly (see fig. 7). Depending on the type of measurement, the voltage switches in an S-like shape (for voltage-controlled IVCs) or jump-like (for current-controlled IVCs) from the nonlinear region of the Abrikosov flux-flow below $\mathrm{V}^{*}$ to a linear regime that resembles the normal state resistance. This behavior has been predicted by Larkin and Ovchinnikov [51]. Recently simulations using time-dependent Ginzburg-Landau theory showed similar IVCs for the high-velocity vortex dynamics in mesoscopic superconductors [59].
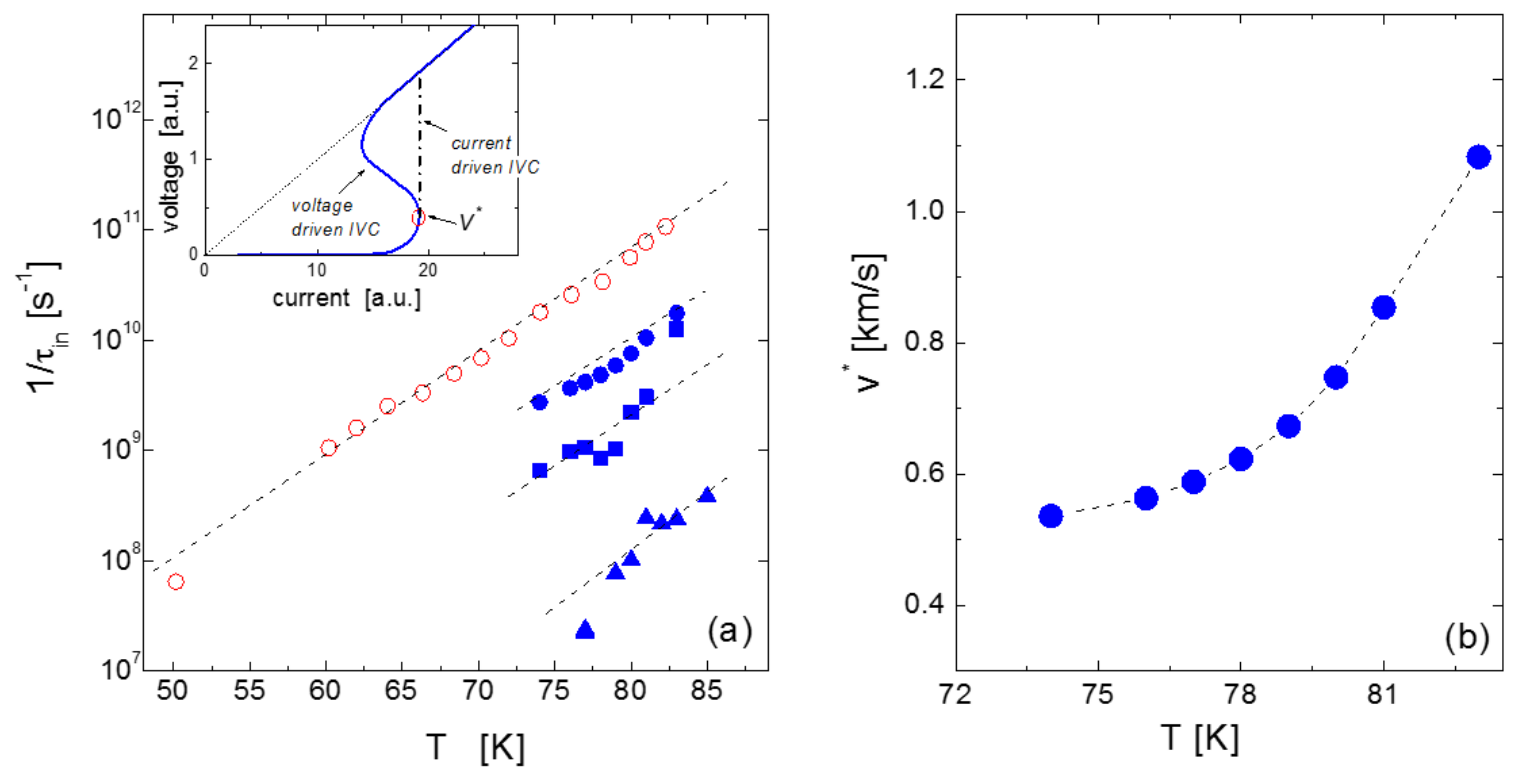

Fig. 7: Inelastic scattering rate (a) and resulting critical vortex velocity (b) as function of temperature for YBCO films obtained from IVC measurements via rapid current ramps (open symbols) [56] and current pulses (solid symbols) [58], respectively. The inset shows a schematic drawing of a voltagecontrolled and current-controlled IVC measured up to voltages $V>V^{*}$. 


\subsubsection{Critical velocity}

Based on Eliashberg's ideas on nonequilibrium effects in superconductors, Larkin and Ovchinnikov predicted that a nonequilibrium distribution and relaxation rate of the normal charge carriers (treated as quasiparticles) develops during the motion of vortices at high velocities [51]. As a result, they expected a discontinuity in the current-voltage characteristic (IVC).

According to the Larkin and Ovchinnikov theory, the viscous damping coefficient at a vortex velocity $v$ is given by [51]

$$
\eta(v)=\eta(0)\left[1+\left(\frac{v}{v^{*}}\right)^{2}\right]^{-1},
$$

with a critical vortex-velocity $v^{*}$

$$
v^{*}=\left[\frac{D \cdot \sqrt{14 \varsigma(3)}}{\pi \tau_{i n}} \cdot \sqrt{1-\frac{T}{T_{c}}}\right]^{1 / 2},
$$

$\tau_{\text {in }}$ denoting the inelastic quasiparticle scattering time, $\zeta(x)$ the Riemann-Zeta function, $D=v_{F} I_{0} / 3$ the quasiparticle diffusion coefficient, $v_{F}$ the Fermi velocity, and $I_{o}$ the electron mean free path. According to this theory, a nonlinear IVC is expected for a critical electric field $E^{*}=v^{*} B$ [51]

$$
J=\frac{E}{\rho_{f f}}\left\{\left(1+\left(\frac{E}{E^{*}}\right)^{2}\right)^{-1}+\gamma \cdot\left(1-\frac{T}{T_{c}}\right)^{1 / 2}\right\}+J_{c},
$$

with $\gamma \approx 1$. This behavior (see fig. 7 ) is observed for conventional superconductors [54,55] as well as for high- $T_{c}$ thin films [56-58]. It appears for instance when the current limit is exceeded in superconducting resistive fault-current limiters. In this case the limiter shows an extremely sharp and sudden voltage peak at the critical electric field $E^{*}$ during the quench of the superconductor at high power [60].

\subsubsection{Phase slip}

The predictions of Larkin and Ovchinnikov [51] refer to extended superconducting systems. The situation changes if vortices move in a mesoscopic system. It is known that flux-transport across a narrow superconducting stripline can occur in form of a phase-slip [52,53]. In this case the phase of the order parameter may periodically slip by $2 \pi$, virtually in a single point. In such a point, the so-called phase-slip center (PSC), the magnitude of the order parameter oscillates between zero and its maximum value. In close similarity to weak links and Josephson junctions, the phase slippage frequency is given by the Josephson relation [52,61]. Originally it was assumed, that phase slip can only occur in wires with a width smaller than the coherence length $\xi$. However, it has been demonstrated that a behavior similar to PSCs may occur also in much wider superconducting striplines. With increasing width of the wire, the PSC may expand to a phase-slip line (PSL), even up to widths much larger than the characteristic length scales $\xi$ or $\lambda$ [62-64].

In contrast to the PSC, the oscillation of the order parameter may not necessarily be uniform along the PSL, they may occur in the form of propagating waves carrying the order parameter singularities across the sample. Such waves have been named kinematic vortices. They have been first predicted in numerical simulations using the 2D time-dependent Ginzburg-Landau equations [65], the experimental evidence for the existence of kinematic vortices is among others reported in Ref. [64]. As such, PSLs 
can be viewed as dynamically created Josephson junctions in homogeneous superconducting films. Therefore the preservation of the macroscopic quantum interference between the two parts of the superconductor defined by the slip line represents a fingerprint of the phase slip mechanism. However, its demonstration for instance via Shapiro steps under microwave irradiation is not trivial.

Besides their superconducting properties, the maximum vortex velocities, which differ by orders of magnitude for the different types of vortices, is of importance for experimental techniques and potential applications. The smallest velocity is observed for classical Abrikosov vortices with a typical maximum speed given by the critical velocity of $\mathrm{v}^{*} \approx 10^{3} \mathrm{~m} / \mathrm{s}$ [56-58]. For $\mathrm{v}>\mathrm{v}^{*}$ the normal core of the Abrikosov vortex collapses. The velocity of kinematic vortices has been estimated to be of the order of $10^{5} \mathrm{~m} / \mathrm{s}$ [64]. The highest velocity is attainable for Josephson vortices, i.e. vortices without a normal core. Their characteristic velocity is of the order of $10^{7} \mathrm{~m} / \mathrm{s}$. A simple explanation of the order and large difference of the different vortex velocities is given by the presence or absence of the normal core and the fact that kinematic vortices have similarities with both Abrikosov and Josephson vortices.

\section{Artificial pinning sites in superconducting electronic devices}

In this section examples of active devices and passive microwave devices will be given that illustrate the role that vortices play in superconducting devices starting with a brief motivation that demonstrates why vortices and vortex motion have to be considered for nearly all superconducting devices.

\subsection{Flux penetration in superconducting electronic devices}

The electronic properties of superconducting devices are usually strongly determined or affected by the presence or motion of vortices. Most superconducting devices will (or have to) operate in magnetic fields strong enough to create large densities of vortices within the device. Generally flux penetrates the superconductor for magnetic fields $B>B_{c 1}$, with the lower critical field $B_{c 1}$. However, the demagnetization effect will lead to a considerable enhancement of the magnetic field at the edge of a superconductor sample. Moreover, calculations of the Gibb's free energy for vortices in thin film devices, approximating the sample geometry by a rectangular cross-section with film thickness $d$ (parallel to the magnetic field direction) much smaller than the lateral dimension $w$, indicate [66] that the first tunnelling of single vortices (e.g., via thermal activated penetration) through the geometrical barrier at the edge of the superconductor is expected to occur already at extremely small fields

$$
B_{T}=\frac{d}{2 w} B_{c 1},
$$

followed by a collective penetration of vortices that takes place at the penetration field

$$
B_{P}=\sqrt{\frac{d}{w}} B_{c 1} .
$$

These predictions are valid for superconducting devices and patterns if $w>d>\lambda$. As a consequence, superconducting films, which represent the major component of most superconducting devices, are usually strongly penetrated by magnetic flux lines (see fig. 8b).

The presences of vortices or their motion will lead to dissipative processes, flux noise, or local modification of the superconducting properties in these devices which, in most applications, means that the performance of the device is diminished. Due to the large anisotropy, the small coherence length and, last but not least, the elevated temperature at which the ceramic superconductors will be operated, these effects are more severe for HTS materials. Ways to avoid the impact of vortices are:

- operation in perfectly shielded environments, 
- reduction of the structures to sizes that do not allow for vortex formation, or

- 'manipulation' of vortex matter.

Since the first two options are in most cases (extremely) costly and/or usually technically difficult or impossible, the manipulation of vortex matter appears to be an ideal solution of this problem. This is one of the motivations for scientific studies on vortex manipulation in superconducting films. Furthermore, the manipulation of vortices is also of interest for the development of (novel) fluxonic concepts. In the following, I will sketch possible routes to improve existing superconducting devices and show concepts for possible novel devices that are based on the manipulation of vortices via artificial pinning sites.
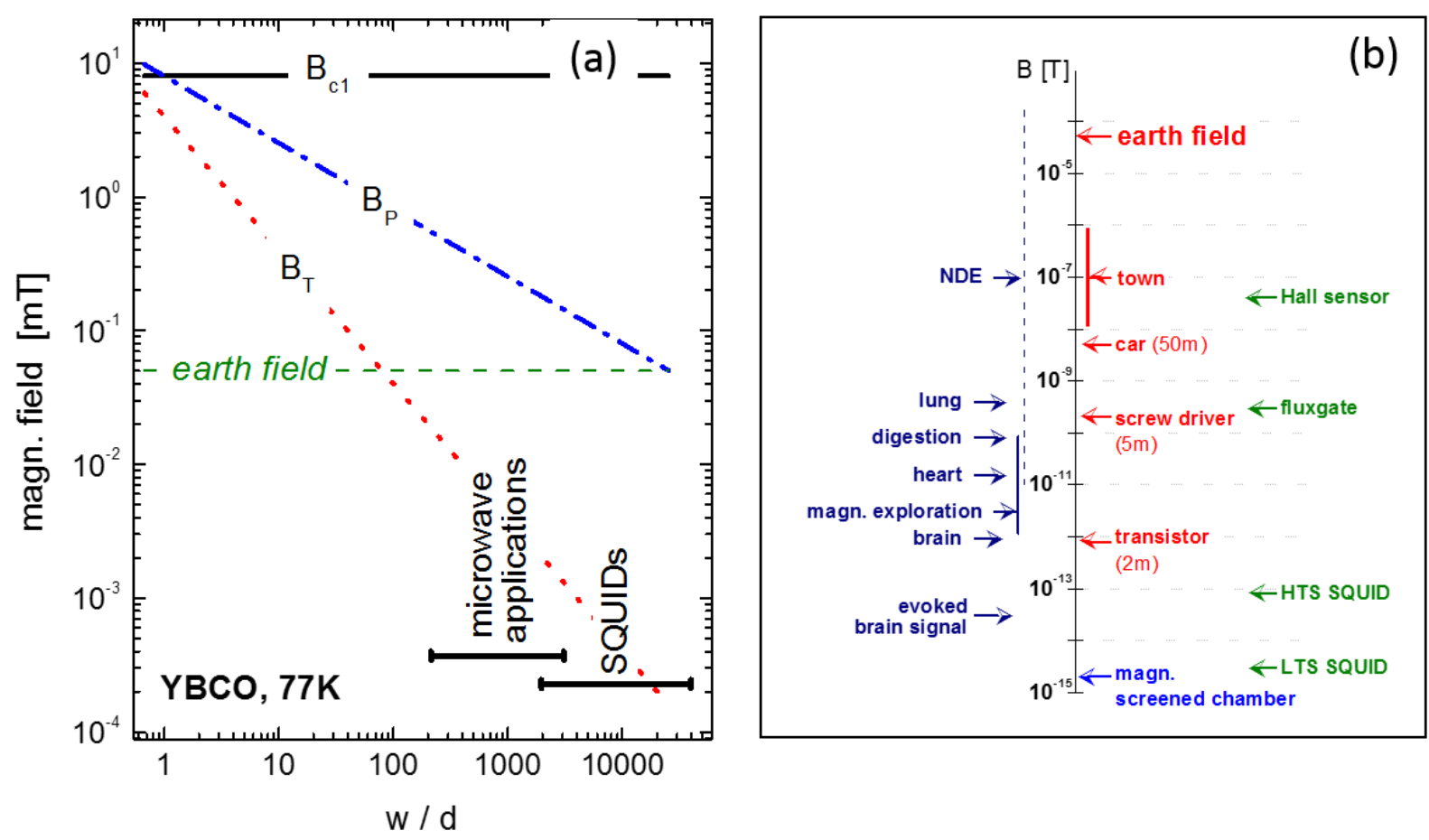

Fig. 8: (a) Critical field $B_{c 1}$, tunnelling field $B_{T}$ and penetration field $B_{P}$ as function of the reduced width calculated for typical YBCO films at 77K. Typical regimes for cryogenic applications are indicated.(b) Comparison of the magnetic-field sensitivity of conventional and superconducting field sensors (right), level of magnetic-field noise (middle), and field sensitivity required for various applications (left).

\subsection{Strategically positioned antidots in Josephson-junction based devices}

\subsubsection{Noise reduction in SQUIDs}

Superconducting quantum interference devices (SQUIDs) represent the most sensitive sensors of magnetic flux or other physical quantities (e.g. currents) that can be transformed into magnetic flux. They are for instance used for non-destructive testing (NDE), geomagnetic applications, biomagnetic or medical applications (e.g. magnetoencephalography). Typical magnetic signals for the different applications are shown in fig. 8 . It demonstrates that the magnetic fields that have to be recorded are 
much smaller (in some cases orders of magnitude smaller) than the magnetic field of other sources like urban noise or even the earth. Therefore it is obvious, that magnetic flux which doesn't stem from the measured object has to considered.

In principle, the SQUID is a deceptively simple device, consisting of (see also fig. 1) a superconducting loop with one (rf-SQUID) or two (dc-SQUID) Josephson junctions, respectively. Magnetic flux threading the superconducting loop leads to a phase difference $\Delta \varphi$ across the Josephson junction which is correlated with the superconducting tunnel current $I_{c}=I_{o} \cdot \sin (\Delta \varphi)=I_{o} \cdot \sin \left(\pi \Phi / \Phi_{o}\right)$ via the $1^{\text {st }}$ Josephson equation (eq. (21) of the tutorial). By reading out the tunnel current (or controlling the tunnel current in a so-called flux-lock-loop), it becomes the most sensitive fluxmeter known with a resolution better than $10^{-6} \Phi_{\text {o }}$.
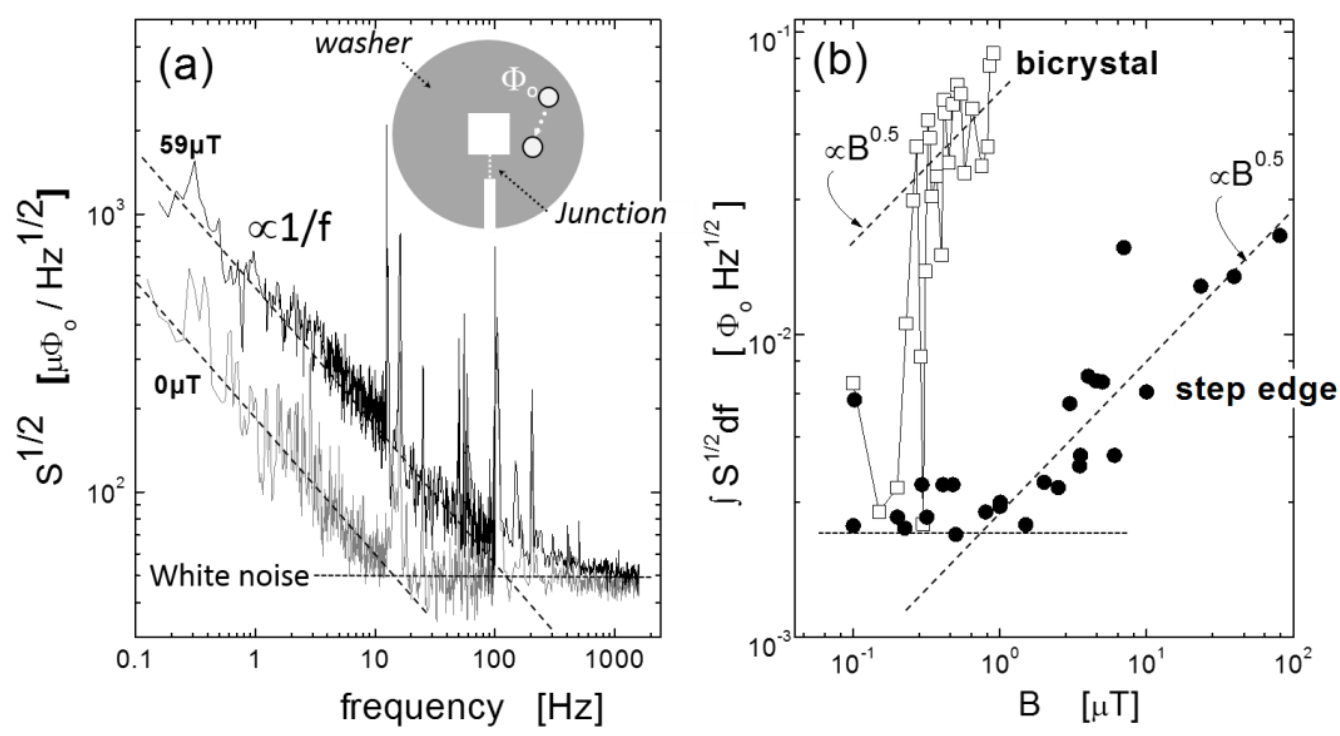

Fig. 9: (a) Spectral noise density for different magnetic fields measured in a HTS step-edge type rfSQUID [11]. The lines idealize the different noise contributions, i.e. the field-dependent $1 / \mathrm{f}$ lowfrequency noise and the field- and frequency-independent white noise. The schematic drawing sketches the geometry of rf-SQUIDs and the problem of a moving vortex in the washer.(b) Magnetic field dependencies of the integral noise $(0.5$ and $10 \mathrm{~Hz})$ of a step-edge and bicrystal Josephson junction according to [11]. The dotted lines represent the theoretical field dependence of the low-frequency noise according to eq. (11).

Generally, the sensitivity of SQUIDs is limited by the frequency dependent noise level of the device. In addition to the contribution of the electronics (usually white noise), in active superconducting devices two different sources are considered to be responsible for the noise. These are the contribution of the active part of the device, which usually consists of one or more Josephson junctions, and the noise coming from the passive component, the superconducting thin film (washer, flux focusser, or flux transformer, depending on the application). The noise mechanisms in Josephson junctions are well understood [67], a reduction of this noise contribution via simple electronic means has been successfully demonstrated [68]. The passive component (e.g. the superconducting film of the loop, washer, or flux transformer) contributes strongly to the low-frequency noise due to by vortex motion in the superconducting film. A nice illustration of this contribution is given by the so called telegraph noise that occurs when a vortex hops between two pinning sites (see fig. 1). In the case of the statistical motion of many vortices (incoherent superposition of many thermally activated microscopic 
fluctuators), a scaling of the spectral noise density $S_{\Phi}$ with frequencies $f$ and the applied magnetic field $B$ is expected

$$
\sqrt{S_{\Phi}(f, B)} \propto \frac{B^{n}}{f^{m}}
$$

with $n=m=0.5$. This so called $1 / f$ noise-spectrum is actually evidence for a distribution of activation energies for the vortex hopping [69]. Typical examples of the spectral noise density and field dependencies of the $1 / f$ integral noise of rf SQUIDs designed for NDE applications are given in fig. 9. The $1 / f$ low-frequency noise increases with magnetic field in agreement with eq. (11), whereas the white noise at high frequencies is more or less field independent.

In section 3.2 it was demonstrated that the low-frequency noise in SQUIDs can be manipulated via antidots. However, it can be seen in fig. 4 and it is evident from theoretical considerations, that regular arrays of antidots lead to noise reduction only at discrete values of the magnetic field (at matching fields) whereas in case of non-commensurability between the vortex and antidot lattice even an increase of the low-frequency noise is observed. Therefore it is better to use only a few, 'strategically positioned' antidots in the superconducting device, which trap only those vortices that attribute strongly to the low-frequency noise (e.g. vortices close to the SQUID hole) and leave the vortex lattice free to arrange itself within the device. Thus, the important issue is to allocate those strategic positions.

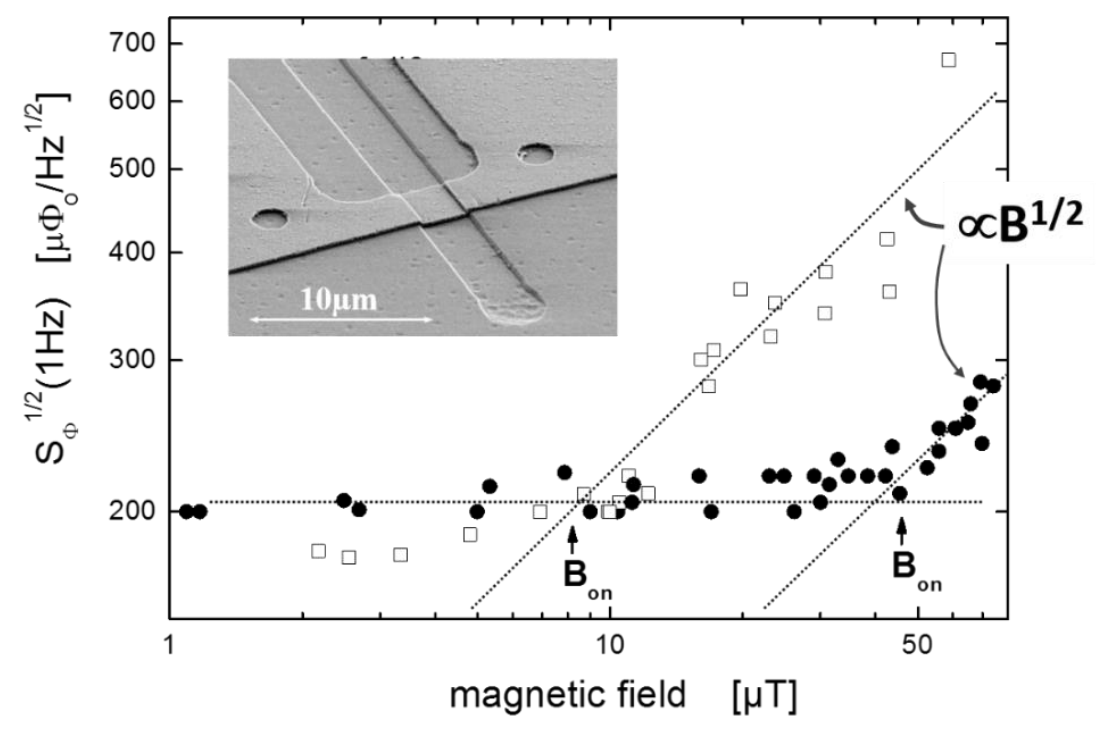

Fig. 10: Spectral noise density at $1 \mathrm{~Hz}(1 / \mathrm{f}$ noise $)$ as a function of the magnetic field for field-cooled measurements for the same rf-SQUID without (open symbols) and with 2 strategically positioned antidots (closed symbols). The inset shows an SEM image of the Josephson junction and the 2 antidots (1.5 $\mu \mathrm{m}$ in diameter). The lines symbolize the white noise and the field dependence according to eq. (11).

An experimental demonstration for noise reduction via strategically positioned antidots is shown in fig. $10[11,12,38]$. Since the largest impact of vortex motion upon the SQUIDs flux noise is expected for vortex motion at a position close to the SQUID hole and close to the Josephson junction, two antidots are positioned on either side of the Josephson junction of an rf-SQUID (see SEM image in fig. 10). The resulting modification of the noise properties is determined in field-cooled experiments on the same SQUID without and with antidots. In these experiments, the SQUID is cooled from the normal to the superconducting state in an applied field $B$ oriented normal to the film surface. A comparison of the spectral noise density in the low-frequency regime $\left(1 / f\right.$ noise) shows, that at low fields $\left(B<B_{\text {on }}\right)$ the 
spectral noise density is field independent, whereas at higher fields $\left(B>B_{\text {on }}\right)$ the spectral noise densities increase linearly with increasing field according to the theoretical expectation $S_{\Phi} \propto B$. However, the transition from field-independent to field-dependent spectral noise density is significantly increased from $B_{o n} \approx 8 \mu \mathrm{T}$ for the measurement without antidots to $B_{o n} \approx 40 \mu \mathrm{T}$ for the configuration with antidots. The increase of the onset field $B_{\text {on }}$ by the arrangement of only 2 strategically positioned antidots is definitely significant. Moreover, it might even be sufficient for a number of SQUID applications in an unshielded environment since the magnetic fields of urban noise and the earth field are typically of the order or smaller than $50 \mu \mathrm{T}$ (see fig. $8 \mathrm{~b}$ ).

\subsubsection{Abricosov-vortex based active devices}

We can invert the effect demonstrated above and utilize the impact of trapped vortices on the signal of a Josephson-type device for superconducting digital devices. Generally, Abrikosov vortices represent extremely small magnetic bits $\Phi_{\circ}$ that can be manipulated for instance by short current pulses and can be detected in different ways by Josephson-type concepts.

A very nice proof of this concept is given in ref. [70] where an Abrikosov vortex based random access memory (AVRAM) memory cell is demonstrated, in which a single vortex is used as an information bit. The basic principle and the operation is illustrated in fig. 11. The AVRAM simply consists of a superconducting line with an antidot and a Josephson-based detector. Vortices are trapped in the antidot and readout via a Josephson detector. The latter could for instance be a Josephson spin-valve or, as sketched in fig. 11, a simple Josephson junction. By applying a small background field (the optimum value depends on the geometry of the device and is typically of the order of Oe for devices of a few $\mu$ m extension) the vorticity of the field trapped by the antidot can be changed by small current pulses between $-\Phi_{0}, 0$, and $+\Phi_{0}$. The magnetic field couples into the Josephson junction and changes the phase and, thus, the Josephson current $J=J_{0} \sin (\Delta \varphi)$. This change of the Josephson current can easily be detected (see fig. 11). This device operates in a similar way to a classical rapid single-flux quantum devices (RSFQ). The use of Abrikosov vortices instead of Josephson vortices (in the case of RSFQ) on the one hand simplifies the device, on the other hand it might restrict the operation regime especially with respect to high frequency applications. Nevertheless, it represents a relatively simple, scalable, low energy, and, fortunately, non-volatile digital device.

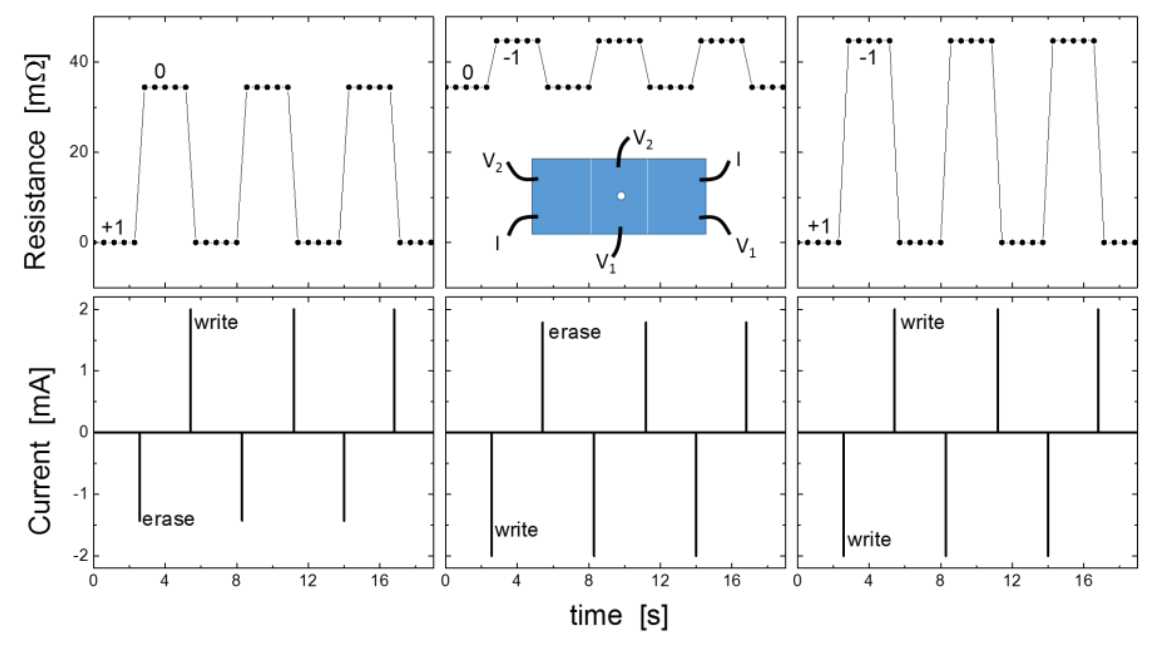

Fig. 11: Demonstration of write and erase operations for an Abrikosov-vortex based random access memory (AVRAM) using a 'strategically' positioned antidot according to ref. [70]. The sketch shows the 
device consisting of a $\mathrm{Nb}$-based stripline equipped with 2 Josephson junctions (dashed white line) and an antidot that is centrally positioned between the Josephson junctions. The device is operated in a moderate field (typically of the order of Oe, depending on the demagnetization factor of the layout). Via sufficiently large current pulses (lower figures) the vorticity of the antidot can be switched between $-1,0$, and 1 (i.e. $-\Phi_{\circ}, 0$, and $+\Phi_{\circ}$ ). The resulting resistance (measured between voltage contacts 1 or 2 applying an ac current with an amplitude larger than the critical current of the junction but much smaller than the pulse current used for write and erase) shows the resulting vortex state (upper figures) for the different switching options.

\subsection{Antidots in Microwave Devices}

Another interesting example represents the use of antidots in superconducting microwave circuitry devices. These have gained increasing interest during the last few years. In particular, coplanar microwave resonators are used in various fields ranging from circuit quantum electrodynamics, quantum information processing, and kinetic inductance particle detection. In all cases high quality factors $Q_{L}=f_{o} / \Delta f$ and low energy losses are essential to these resonators. In some cases, these devices have to operate in (moderate) magnetic fields. In these cases vortices and their motion strongly affect the performance of the device. An obvious solution is given by the use of artificial pinning sites, especially antidots.

The basic components of microwave devices are impedance matched stiplines. The microwave current is strongly peaked at the edge of the conductor. For example, the current densities in the central conductor of a coplanar microwave devices can be approximated by [71]:

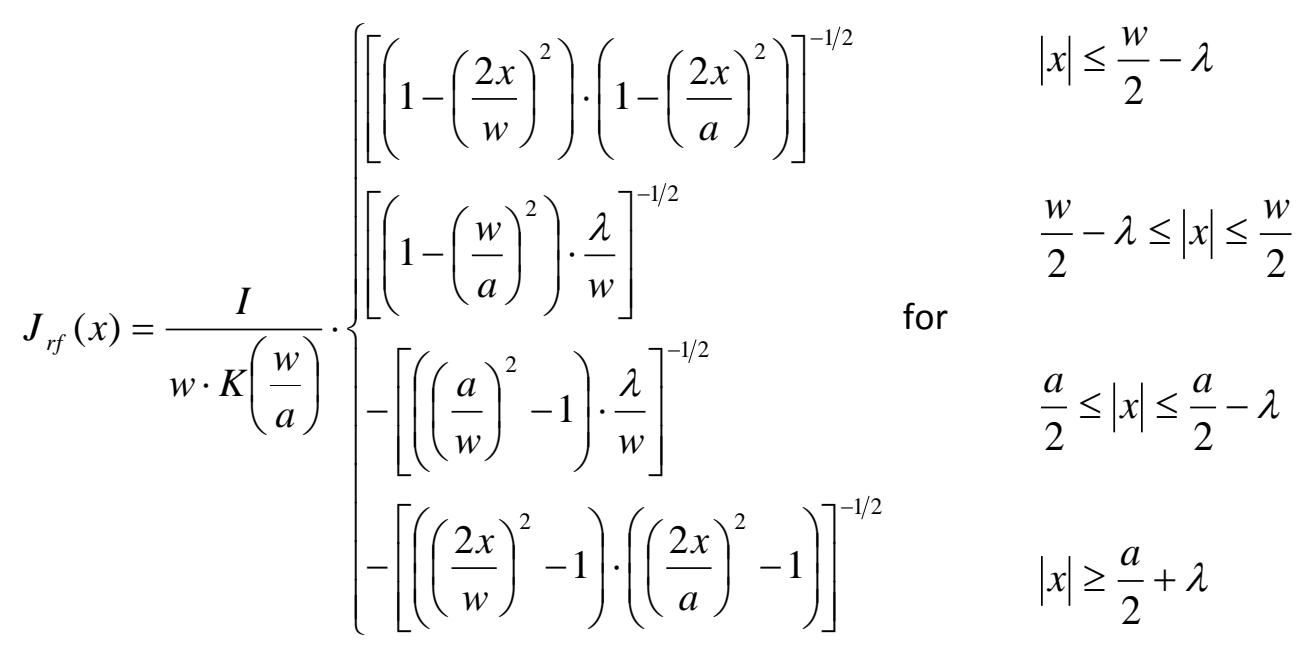

with $w$ representing the width of the stripline, $a$ the distance between the ground planes, and $K$ the complete elliptical integral of the first kind. A sketch of the cross-section of the structure and the resulting microwave current distribution is given in fig. 12a. Since vortices will penetrate at the edge of the superconducting stripline, the microwave losses are strongly affected by any modification of the applied magnetic field (see sketches in fig. 12b).

This is demonstrated by comparing field-cooled ( $\mathrm{fc}$ ) and zero-field cooled (zfc) measurements of the power handling capability of microwave resonators that are exposed to small magnetic fields (fig. 12c) [16]. In fc experiments the resonator is cooled to the superconducting state in the applied magnetic field (i.e., the vortex distribution is expected to be homogeneous), whereas in $\mathrm{zfc}$ experiments the resonator is cooled to the superconducting state in zero-field and only then the magnetic field is applied in the superconducting state. In the latter case, vortices have to penetrate from the edge of the superconductor, leading to an inhomogeneous distribution of vortices. Thus, the expected vortex distribution is different for $\mathrm{fc}$ and $\mathrm{zfc}$ experiments (see sketches in fig. 12c). 


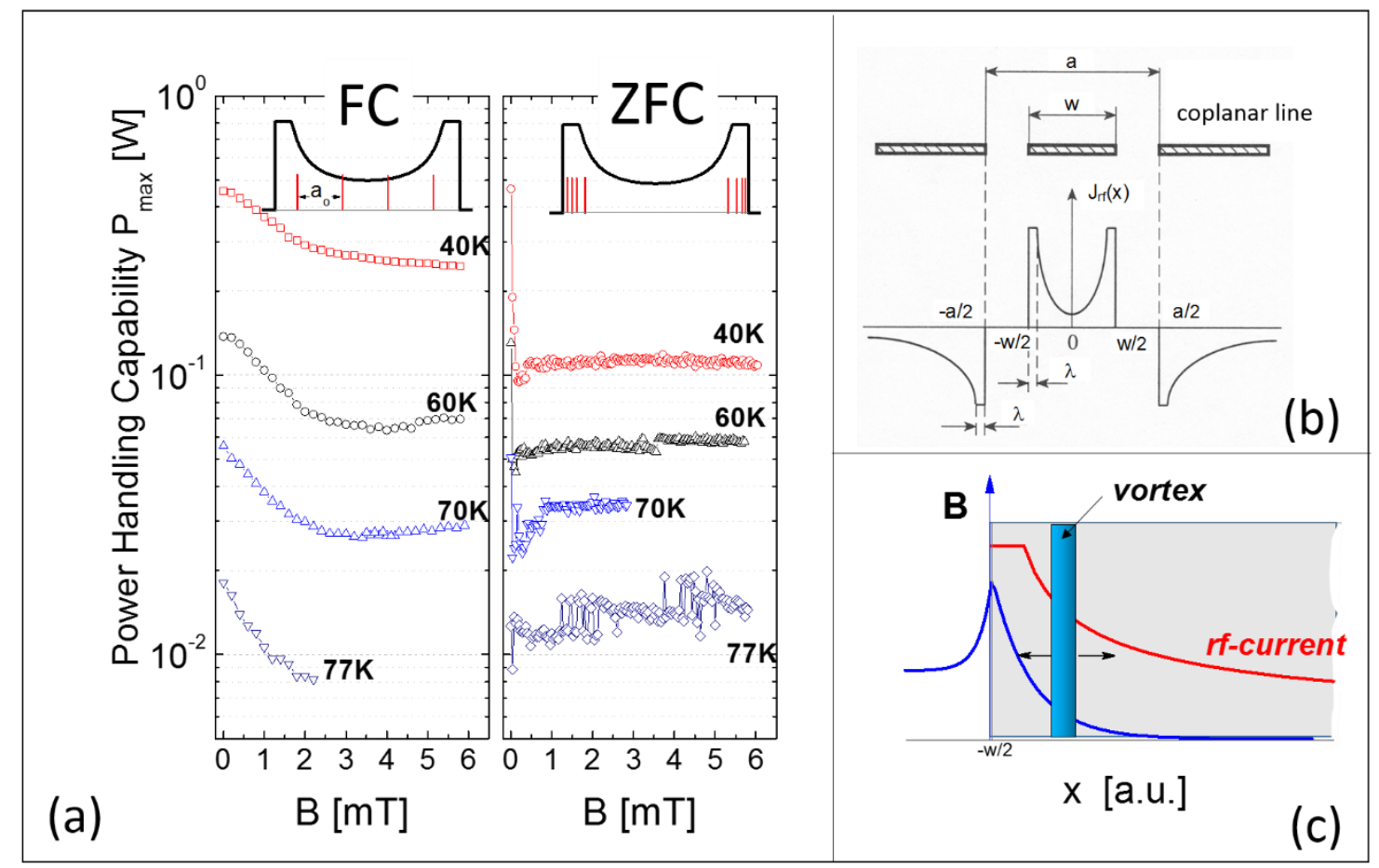

Fig. 12: Schematic drawing of (a) the microwave current distribution $J_{r f}$ in a coplanar superconducting lines according to eq.(31) and (b) the microwave current and magnetic field distribution at the edge of a superconducting stripline. (c) Field dependence of the power handling capability of a coplanar YBCO resonator for field-cooled and zero-field cooled experiments at different temperatures [16]. The power handling is characterized by the degradation of the loaded quality factor $Q_{L}=f_{0} / \Delta f$, i.e., $P_{\max }$ is defined by the condition $Q_{L}\left(P_{\max }\right)=0.8 Q_{L}\left(P_{o}\right)$. The sketches in (c) indicate the different vortex distribution in field cooled and zero-field cooled experiments and the microwave current distribution in the central conductor of the coplanar microwave resonator.

The first attempts to improve the performance of HTS microwave resonators exposed to magnetically unshielded environment via antidots were published in $[16,72]$. A systematic study of the impact of antidots followed for $\mathrm{Nb}$-based microwave resonators $[17,18]$. The main results of these experiments on HTS and $\mathrm{Nb}$ resonators are illustrated in fig. 13:

(i) The performance of the resonator operating in an applied magnetic field of up to a few $\mathrm{mT}$ can be improved by antidots. This holds for the quality factor (see fig. 13) and the frequency stability of the resonator.

(ii) The improvement is large for fc-experiments where we expect a homogeneous distribution of the vortices in the stripline. For zfc experiments this effect is much smaller. The reason is that vortices or antivortices are concentrated at the edge of the stripline for increasing field or decreasing field, respectively (see sketches in fig. 12) [16]. Since this is the area of large microwave current, this leads to a strong decrease of the performance and a reduced impact of the antidots. 
(iii) The kink in the field dependence of the quality factor (see inset of fig. 13) reveals an interesting effect that is connected to the penetration of the flux into the center of the stripline. It actually allows one to tune the performance of the resonator by appropriate field sweeps [16].

The demonstrated results are very encouraging. In the first experiments the position of the antidots was optimized but the antidots were still quite large $[16,17,72]$, whereas in the later experiments the size of the antidots was minimized [18]. Further improvement might be possible if both the size of the antidots can be reduced even further and the position of the perforation can be optimized.

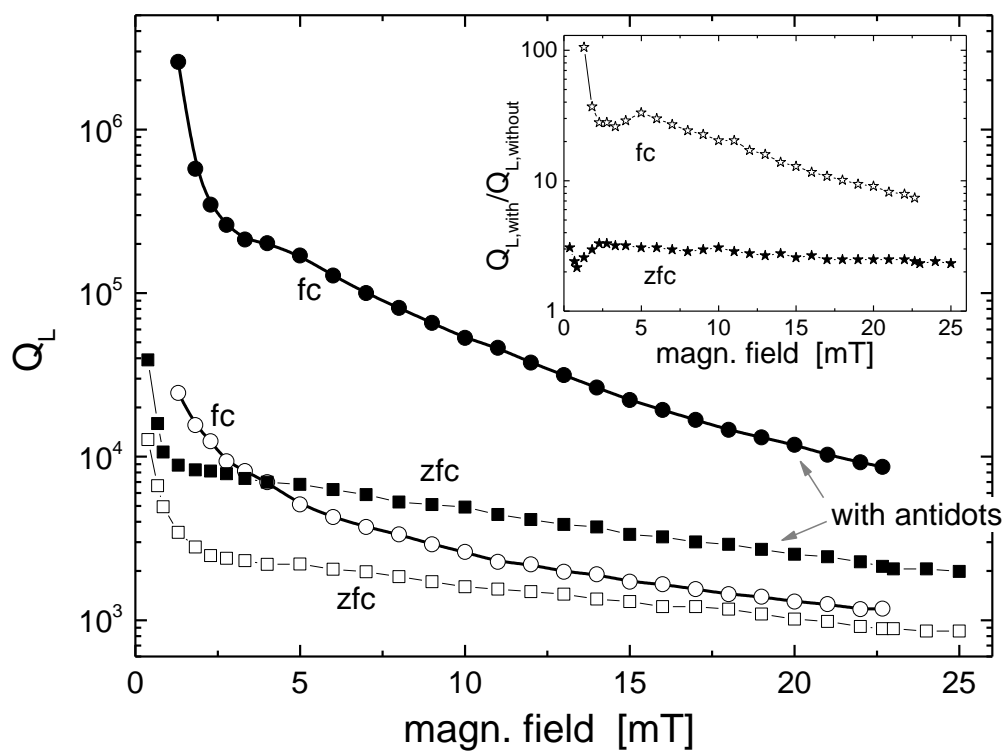

Fig. 13: Loaded quality factor at the fundamental mode for a $60 \mu \mathrm{m}$ wide coplanar $\mathrm{Nb}$ based resonator with (solid symbols) and without (open symbols) antidots at $4.2 \mathrm{~K}$ for field-cooled (circles) and zerofield cooled (squares), data adopted from [18]. In this case the central line of the resonator is completely covered with a quasihexagonal array of antidots of submicrometer antidot size and an antidot density of approx. $1.65 \mu \mathrm{m}^{-2}$.

\subsection{Concepts for Fluxonic Devices}

In the previous sections, strategies to improve existing superconducting devices by manipulating vortices were introduced. However, the field of vortex manipulation by micro- or nanostructures in superconducting films is much more colorful. Different feasible concepts are indicated in fig. 14. For instance, vortices might be generated, guided and trapped by slits, or small and large antidots, respectively. By appropriate magnetic field variations or using the current curls created by a slit at opposing edges of a stripline, even vortices and antivortices can be created and manipulated. The operation of some of these components has been demonstrated for low- $\mathrm{T}_{\mathrm{c}}$ films (e.g., $\mathrm{Nb}, \mathrm{Pb}$ or $\mathrm{Al}$ films) or their impact upon magnetic flux has been visualized by magnetooptic experiments $[74,74]$. An interesting option is suggested by the use of asymmetric antidots that could lead to a directed vortex motion, the so called ratchet effect. 


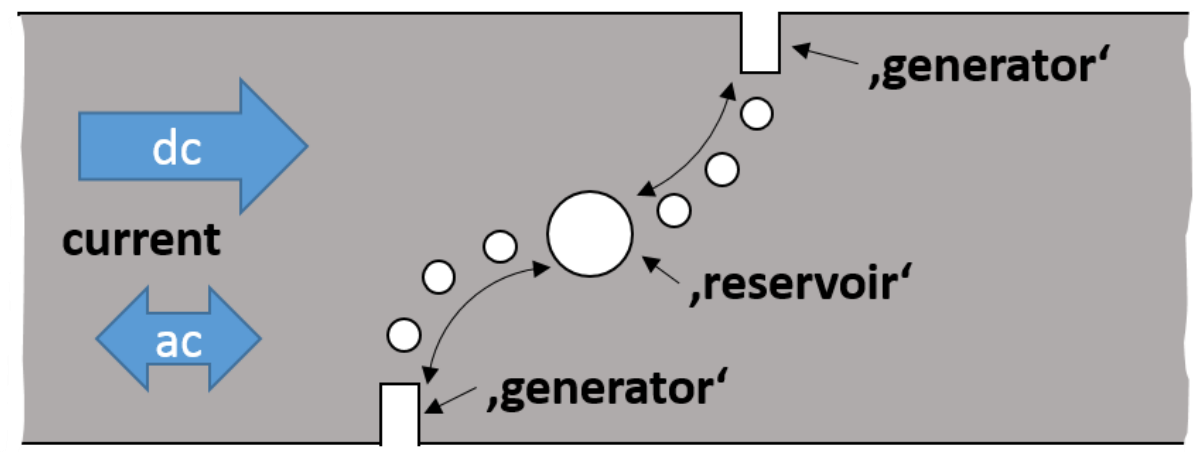

Fig. 14: Sketch of different nano- and microstructure components for vortex manipulation in HTS films that might lead to novel fluxonic applications. Vortices and antivortices (vortices with inverted field component) can be generated by curls in the dc or microwave current caused by slits at opposite edges of a stripline, the 'generators' for vortex formation. Guidance, trapping, and annihilation are achieved by antidots of different size. Annihilation of vortices would be possible in the case of guidance of a vortex and an antivortex towards the same trapping site, the 'reservoir'.

Generally, ratchets are formed from spatially asymmetric confining potentials. They can rectify oscillatory driving forces and generate directed motion. Ratchet scenarios were already considered by Feynman in his lecture notes in 1963 [75] and are related to earlier problems in thermodynamics studied by Smoluchowski in 1912 [76]. Ratchets represent a major component of particle transport in nanoscale systems, both in solid-state systems and in biology. Ratchets in biological systems (e.g., biomolecular motors) can be found in nature, including the kinesin and dynein proteins that provide transport functions within the cell [77]. Ratchets can be produced by biomolecular engineering, e.g., a molecular walker constructed from strands of DNA [78] and controlled motion of kinesin-driven microtubules along lithographically patterned tracks have been demonstrated recently [79]. Advances in nanofabrication made it feasible to develop and investigate ratchets formed from solid-state systems involving electronic devices or microfluidics. Such devices can be used (i) as analog systems for modeling biomolecular motors, (ii) to understand novel particle transport at the nanoscale or (iii) to develop new devices for application. One advantage of solid-state nanofabrication is the possibility to tailor the ratchet potential and to control driving parameters, temperature or other process parameters. Implementations of ratchets in electronic devices have been demonstrated recently, e.g., the use of asymmetric structures of electrostatic gates above a 2D electron gas [80] and arrays of Josephson junctions with asymmetric critical currents [81].

Vortices in superconductors form an ideal system for exploring ratchet phenomena. The control of vortex dynamics via micro or nanostructures allows for the tailoring of vortex confining potentials. One approach for controlling and rectifying vortex motion in superconductors involves the use of arrays of antidots. Vortex ratchet effects obtained by various arrangements of antidots have recently been demonstrated for low- $T_{c}$ films [82-85] and high- $T_{c}$ films [6]. The asymmetric pinning potential is achieved by asymmetrically shaped antidots, combinations of antidots of different sizes, the asymmetric arrangement of symmetric antidots or even mixing of dc and ac currents, respectively. 


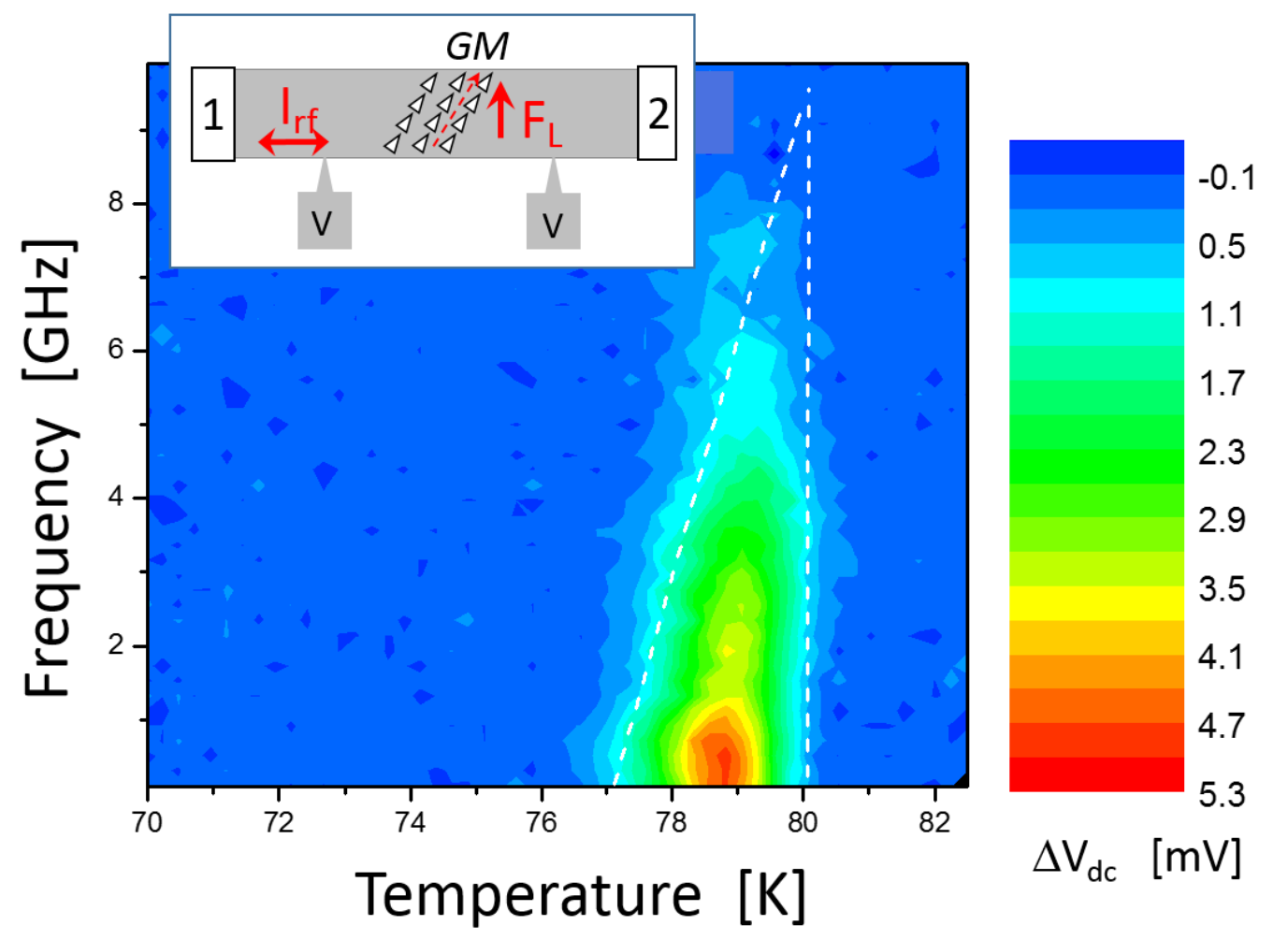

Fig. 15: Demonstration of the ratchet effect in HTS microwave devices. Schematic drawing of a YBCO ratchet based on rows of triangular shaped antidots (inset) and contour plot of the change of the dc voltage signal $\Delta V_{\text {longitudinal }}=V_{\text {longitudinal }}(-10 \mathrm{dBm})-\mathrm{V}_{\text {longitudinal }}(-50 \mathrm{dBm})$ as a function of temperature and applied microwave frequency. The microwave current is applied via ports 1 and 2, the vortices are rectified by rows of triangular antidots and the dc longitudinal is recorded at contacts $V$. The change of the rectified longitudinal voltage signal due to the microwave power demonstrates rectified vortex motion for temperatures close to $T_{c}$ (high vortex mobility) and up to frequencies of about $10 \mathrm{GHz}$.

Fig. 15 shows an example of a HTS ratchet that also illustrates the potential use of ratchets in microwave applications. The design of the device (fig. 15a) is similar to the design used for the demonstration of guided vortex motion (see fig. 6), except for the fact, that asymmetric (here, triangular shaped) antidots are used. The tilting of the direction of antidot rows with respect to the Lorentz force allows for simultaneous measurements of a longitudinal and Hall signal in the case of guided vortex motion. In the case of the rectification of the vortex motion (ratchet effect) a microwave driving current would be transformed into a directional motion of vortices and, thus, a dc voltage at the Hall and longitudinal voltage pads. Fig. 15b shows a contour plot of the dc voltage (only the longitudinal voltage is shown) obtained in a typical experiment. In order to improve the resolution of the measurement, a reference signal taken at low microwave power $(-50 \mathrm{dBm})$ is subtracted from the signal recorded at large microwave power $(-10 \mathrm{dBm})$.

The experiment demonstrates that HTS vortex ratchets can operate, although in a restricted temperature regime close to $T_{c}$ and a frequency regime up to about $10 \mathrm{GHz}$. An obvious restriction of the operation regime is imposed by the limited microwave power used in the experiment. Close to $T_{c}$, the pinning force is small and vortices move easily. At lower temperatures, the pinning force and viscosity increase and, as a consequence, larger microwave power is needed to move vortices between the antidots. This restricts the temperature regime for operation to temperatures close to $T_{c}$. Nevertheless, the temperature regime is still quite large compared to the operation regimes of low- $T_{c}$ 
films, where ratchet effects are typically present in the range $0.995<T / T_{c}<1$. Whether the frequency limit (approx. $10 \mathrm{GHz}$ ) is a fundamental limit for the motion of Abrikosov vortices in HTS devices is not clarified up to now. Experiments performed at larger microwave power or using smaller antidot spacings might prove this in the future.

The principle of the vortex ratchet is one of the most interesting components for basic analysis and maybe also in the future for application of vortex manipulation in superconducting films and devices. Its operation has been demonstrated. They might be used as a converter (microwave-to-dc), filters, or as a component in more complex microwave devices that could offer interesting and novel properties.

\section{Conclusions}

Vortex matter in superconducting films and devices is not only an interesting topic for basic research but plays a substantial role in the applications of superconductivity in general. In most electronic applications, magnetic flux will penetrate the superconductor. Magnetic flux and flux motion affect the performance of superconducting devices. For instance, the reduction of the sensitivity in SQUIDs or the power-handling capability in microwave devices, or the increase of the error rate in logic devices is a consequence of the presence of flux and flux motion in these devices. Guidance and trapping of vortices can reduce or even prevent this effect.

Moreover, vortex manipulation is not only a useful tool to avoid degradation of superconducting device properties, it can also be used to analyze and understand novel and interesting physical properties and to develop new concepts for superconductor applications. Various concepts for vortex manipulation are sketched. The advantage of the use of micro- and nanopatterns (especially, antidots) for guiding and trapping of vortices is discussed and experimental evidence of vortex guidance and vortex trapping by various arrangements of antidots is given.

Thus, the vortex state of matter appears to be very important in applications of superconductivity and requires further investigation. A better understanding will clearly lead to an improvement of the performance of superconductor components, such as reduced noise, better power handling capability, or improved reliability. Furthermore, it promises deeper insight into the basic physics of vortices and vortex matter, especially at high frequencies. The use of different experimental techniques in combination with micro- or even nanopatterning of high- $T_{\mathrm{c}}$ superconducting film might pave the way towards strategic manipulation of vortices and, thus, a route towards interesting and innovative fluxonic effects and device concepts.

\section{Acknowledgment}

The author likes to acknowledge the experimental work of A. M. Castellanos, P. Selders, M. Pannetier, R. Wijngaarden, A. Pruymboom, P. Dymachevski, and P. Lahl. 


\section{References}

1: see for instance R. Prozorov, M. Kończykowski, M. A. Tanatar, A. Thaler, S. L. Bud'ko, P. C. Canfield,V. Mishra, and P. J. Hirschfeld, Phys. Rev. X 4, 041032 (2014)

2. A. Pruymboom, P. H. Kes, E. van der Drift, S. Radelaar, Appl. Phys. Lett. 52, 662 (1988); A. Pruymboom, P. H. Kes, E. van der Drift, S. Radelaar, Phys. Rev. Lett. 60, 1430 (1988)

3. R. Besseling, Thesis, University of Leiden (2001)

4. A. M. Castellanos, R. Wördenweber, G. Ockenfuss, A. v.d. Hart, K. Keck, Appl. Phys. Lett. 71, 962 (1997)

5. R. Wördenweber, A. M. Castellanos, P. Selders, Physica C 332, 27 (2000)

6. R. Wördenweber, P. Dymashevski, and V. R. Misko, Phys. Rev. B 69, 184504 (2004)

7. F. Laviano, G. Ghigo, E. Mezzetti, E. Hollmann and R. Wördenweber, 2010, Control of the vortex flow in microchannel arrays produced in YBCO films by heavy-ion lithography, Physica C 19, 844847

8. M. P. González, E. Hollmann and R. Wördenweber, J. Appl. Phys. 102, 063904 (2007)

9. N. Kokubo, Phys. Rev. Lett. 88, 247004 (2002)

10. K. Yu, T. W. Heitmann, C. Song, M. P. DeFeo, B. L. T. Plourde, M. B. S., Hesselberth, and P. H. Kes, Phys. Rev. B 76, 220507 (2007)

11. P. Selders, R. Wördenweber, Appl. Phys. Lett. 76, 3277 (2000)

12. R. Wördenweber, P. Selders, Physica C 366, 135 (2002)

13. G. R. Berdiyorov, M. V. Milošević, and F. M. Peeters, 'Qualitative modification of the pinning force due to vortex-cavity adherence in perforated superconducting films', unpublished

14. G. S. Mkrtchyan and V. V. Shmidt, Sov. Phys. JETP 34, 195 (1972)

15. A. I. Buzdin and D. Feinberg, Physica C 256, 303 (1996)

16. P. Lahl, R. Wördenweber, Appl. Phys. Lett. 81, 505 (2002)

17: D. Bothner, T. Gaber, M. Kemmler, D. Koelle, and R. Kleiner, Appl. Phys. Lett. 98, 102504 (2011)

18: D. Bothner, C. Clauss, E. Koroknay, M. Kemmler, T. Gaber, M. Jetter, M. Scheffler, P. Michler, M. Dressel, D. Koelle, and R. Kleiner, Appl. Phys. Lett. 100, 012601 (2012)

19. M. Baert, V. V. Metlusko, R. Jonckheere, V. V. Moshchalkov, Y. Bruynseraede, Phys. Rev. Lett. 74, 3269 (1995)

20. E. Rosseel, M. van Bael, M. Baert, R. Jonckheere, V. V. Moshchalkov, Y. Bruynserade, Phys. Rev. B 53, R2983 (1996)

21. V. V. Metlushko, M. Baert, R. Jonckheere, V. V. Moshchalkov, Y. Bruynserade, Sol. St. Comm. 91, 331 (1994)

22. V. V. Moshchalkov, M. Baert, E. Rosseel, V. V. Metlushko, M. J. van Bael, Y. Bruynserade, Physica C 282, 379 (1997)

23. V. V. Moshchalkov, M. Baert, V. V. Metlushko, E. Rosseel, M. J. van Bael, K. Temst, Y. Bruynserade, R. Jonckheere, Phy. Rev. B 57, 3615 (1998)

24. L. van Look, E. Rosseel, M. J. van Bael, K. Temst, V. V. Moshchalkov, Y. Bruynseraede, Phys. Rev. B 60, R6998 (1999)

25. A. N. Lykov, Sol. St. Comm. 86, 531 (1993)

26. K. Harada, O. Kamimura, H. Kasai, T. Matsuda, A. Tonomura, V. V. Moshchalkov, Science 274, 1167 (1996)

27. A. Bezryadin, Y. N. Ovchinikov, and B. Pannetier, Phys. Rev. B 53, 8553 (1996); A. Bezryadin and B. Pannetier, J. Low Temp. Phys. 102, 73 (1996)

28. V. V. Metlushko, U. Welp, G. W. Crabtree, Zhao Zhang, S. R. J. Brueck, B. Watkins, L. E. DeLong, B. Ilic, K. Chung, P. J. Hesketh, Phys. Rev. B 59, 603 (1999)

29. V. V. Metlushko, U. Welp, G. W. Crabtree, R. Osgood, S. D. Bader, L. E. DeLong, Zhao Zhang, S. R. J. Brueck, B. Ilic, K. Chung, P. J. Hesketh, Phys. Rev. B 60, R12585 (1999)

30. R. Wördenweber, E. Hollmann, J. Schubert, R. Kutzner, and Ajay Kumar Ghosh, Appl.Phys. Lett. 94, 202501 (2009).

31. H. Nordborg and V. M. Vinokur, Phys. Rev. B 62, 12408 (2000) 
32. C. P. Bean and J. D. Livingston, Phys. Rev. Lett. 12, 14 (1964)

33. R. Surdeanu, R. J. Wijngaarden, R. Griessen, J. Einfeld, and R. Wördenweber, Europhys. Lett. 54, $682(2001)$

34. V.V. Moshchalkov, M. Baert, V.V. Metlushko, E. Rosseel, M.J. van Bael, K. Temst, R. Jonckheere, Y. Bruynserade, Phys. Rev. B 54, 7385 (1996)

35: Misko V, Savel'ev S and Nori F 2005 Phys. Rev. Lett. 95177007

36: Kemmler M, Gürlich C, Sterck A, Pöhler H, Neuhaus M, Siegel M, Kleiner R and Koelle D 2006 Phys. Rev. Lett. 97147003

37: D Bothner, R Seidl, V R Misko, R Kleiner, D Koelle and M Kemmler, Unusual commensurability effects in quasiperiodic pinning arrays induced by local inhomogeneities of the pinning site density, Supercond. Sci. Technol. 27 (2014) 065002

38. P. Selders, A. M. Castellanos, M. Vaupel, and R. Wördenweber, Appl. Supercond. 5, 269 (1998)

39. M. Pannetier, R. J. Wijngaarden, I. Fløan, J. Rector, R. Griessen, P. Lahl, and R. Wördenweber, Phys. Rev. B 67, 212501 (2003)

40. A. Lukashenko, A. V. Ustinov, A. P. Zhuravel, E. Hollmann, and R. Wördenweber, J. Appl. Phys. 100, 023913 (2006).

41. S. J. Hagen, C. J. Lobb, R. L. Greene, M. G. Forrester and J. H. Kang, Phys. Rev. B 41, 11630 (1990).

42. S. J. Hagen, A. W. Smith, M. Rajeswari, J. L. Peng, Z. Y. Li, R. L. Greene, S. N. Mao, X. X. Xi, S. Bhattacharya, Qi Li, and C. J. Lobb, Phys. Rev. B 47, 1064 (1993).

43. P. Ao, J. Phys. Condens. Matter 10, L677 (1998).

44. H.J. Jensen, P. Minnhagen, E. Sonin, and H. Weber, Europhys. Lett. 20, 463 (1992).

45. R.A. Ferrell, Phys.Rev. Lett. 68, 2524 (1992).

46. A.van Otterlo, M. Feigel'man, V. Geshkenbein, and G. Blatter, Phys. Rev. Lett. 75, 3736 (1995).

47. D.I. Khomskii and A. Freimuth, Phys. Rev. Lett. 75, 1384 (1995).

48. N.B. Kopnin, Phys. Rev. B 54, 9475(1996).

49. J. Kolacek and P. Vasek, Physica C 336, 199 (2000).

50. R. Wördenweber, J.S.K. Sankarraj, P. Dymashevski, and E. Hollmann, Physica C 434, 101 (2006).

51. A. I. Larkin, Yu. N. Ovchinnikov, Zh. Eksp. Teo. Fiz. 68, 1915 (1975) and Sov. Phys.-JETP 41, 960 (1976)

52: W.J. Skocpol, M.R. Beasley, and M. Tinkham, J. Low. Temp. Phys. 16, 145 (1974)

53: A.M. Kadin, L.N. Smith, and W.J. Skocpol, J. Low Temp. Phys. 38, 497 (1980)

54. L. E. Musienko, I. M. Dmitrenko, und V. G. Volotskaya, Pis'ma Zh. Eksp. Teor. Fiz. 31, 603 (1980) and JETP Lett. 31, 567 (1980)

55. W. Klein, R. P. Huebener, S. Gauss und J. Parisi, J. Low. Temp. Phys. 61, 413 (1985)

56. S. G. Doettinger, R. P. Huebener, R. Gerdemann, A. Kühle, S. Anders, T. G. Träuble, und J. C. Villégier, Phys. Rev. Lett. 73, 1691 (1994) and Physica C 235-240, 3179 (1994)

57. S.G. Doettinger, R.P. Huebener, and A. Kühle, Physica C 251, 285 (1995)

58. P. Lahl and R. Wördenweber, Inst. Phys. Conf. Ser. 158, 77 (1997)

59: G. Grimaldi, A. Leo, P. Sabatino, G. Carapella, A. Nigro, S. Pace, V. V. Moshchalkov, and A. V. Silhanek, Phys. Rev. B 92, 024513 (2015)

60. R. Wördenweber, U. Krüger, J. Schneider, R. Kutzner, and G. Ockenfuß, Inst. of Phys. Conf. Ser. 148, 619 (1995)

61: V. I. Kuznetsov and V. A. Tulin, Sov. Phys. JETP 86, 1364 (1998)

62. A. Weber and L. Kramer, J. Low Temp. Phys. 84, 289 (1991)

63. J. Schneider, H. Kohlstedt, and R. Wördenweber, Appl. Phys. Lett. 63, 2426 (1993)

64: A.G. Sivakov, A.M. Glukhov, and A. N. Omelyanchouk, Y. Koval, P. Müller, and A.V. Ustinov, Phys. Rev. Lett. 91, 267001 (2003)

65. A. Andronov, I. Gordion, V. Kurin, I. Nefedov, and I. Shereshevsky, Physica C 213, 193 (1993)

66. E. Zeldov, A. I. Larkin, V. B. Geshkenbein, M. Konczykowski, D. Majer, B. Khaykovich, V. M. Vinokur, H. Shtrikman, Phys. Rev. Lett. 731428 (1994)

67. R. Gross, B. Mayer, Physica C 180, 235 (1991); M. Kawasaki, P. Chaudhari, and A. Gupta, Phys. Rev. Lett. 68, 1065 (1992); A. Marx and R. Gross, Appl. Phys. Lett. 70, 120 (1997); A. H. Miklich, J. Clarke, M. S. Colclough, K. Char, Appl. Phys. Lett. 60, 1899 (1992) 
68. R. L. Forgacs, A. F. Warwick, Rev. Sci. Instrum. 38, 214 (1967); V. Foglietti, W. J. Gallagher, M. B. Ketchen, A. W. Kleinsasser, R. H. Koch, S. I. Raider, and R. L. Sandstrom, Appl. Phys. Lett. 49, 1393 (1986); R. H. Koch, J. Clarke, W. M. Goubau, J. M. Martinis, C. M. Pegrum, and D. J. van Harlingen, J. Low Temp. Phys. 51, 207 (1983); R. H. Koch, W. Eidelloth, B. Oh, R. P. Robertazzi, S. A. Andrek, and W. J. Gallagher, Appl. Phys. Lett. 60, 507 (1992); A. H. Miklich, D. Koelle, E. Dantsker, D. T. Nemeth, J. J. Kingston, R. F. Kromann, and J. Clarke, IEEE Trans. Appl. Supercond. 3, 2434 (1993); M. Mück, C. Heiden, and J. Clarke, J. Appl. Phys. 75, 4588 (1994)

69. P. Dutta, P. Dimon, and P. M. Horn, Phys. Rev. Lett. 60, 646 (1979); P. Dutta and P. M. Horn, Rev. Mod. Phys. 53, 497 (1981)

70: T. Golod, A. lovan, and V.M. Krasnov, Nat. Comm. 6, 8628 (2015)

71. I. B. Vendik, O. G. Vendik, and D. I. Kaparkov, Superconductor Microwave Technology, Part II: Superconducting Microwave Circuits, Chalmers University of Technology, Report No 24 (ed. Kollberg E) ISSN 1103-4599, ISRN CTH-MVT-R-24--SE (1996)

72: R. Wördenweber, High-Tc films: From natural defects to nanostructure engineering of vortex matter, 2010, in Nanoscience and Engineering in Superconductivity, edts. Moshchalkov, V.; Wördenweber, R.; Lang, W., Series: NanoScience and Technology, ISBN: 978-3-642-15136-1, Springer, p. 25-80

73. M. Baziljevich, T. H. Johansen, H. Bratsberg, Y. Shen and P. Vase, Appl. Phys. Lett. 69, 3590 (1996)

74. V. V. Yurchenko, R. Wördenweber, Yu. M. Galperin, D. V. Shantsev, J. I. Vestgården and T. H. Johansen, Physica C 437-438, 357 (2006)

75. R. P. Feynman, R. B. Leighton, and M. Sands, The Feynman Lectures on Physics. Addison-Wesley, Reading, MA, 1963, vol. I.

76. M. Smoluchowski, Phys. Z. 13, 1069 (1912)

77. E. Kay, D. Leigh, and F. Zerbetto, Angew. Chem. Int. 46, 72 (2007)

78. T. Omabegho, R. Sha, and N. C. Seeman, Science 324, 67 (2009)

79. Y. Hiratsuka, T. Tada, K. Oiwa, T. Kanayama, and T. Uyeda, Biophys. J. 81, 1555 (2001)

80. H. Linke, T. E. Humphrey, A. Löfgren, A. O. Sushkov, R. Newbury, R. P. Taylor, and P. Omling, Science 286, 2314 (1999)

81. D. E. Shalóm and H. Pastoriza, Phys. Rev. Lett. 94, 177001 (2005)

82. B. Y. Zhu, F. Marchesoni, V. V. Moshchalkov, and F. Nori, Phys. Rev. B 68, 014514 (2003)

83. J. Van de Vondel, C. C. de Souza Silva, B. Y. Zhu, M. Morelle, and V. V. Moshchalkov, Phys. Rev. Lett. 94, 057003 (2005)

84. C. C. de Souza Silva, J. Van de Vondel, B. Y. Zhu, M. Morelle, and V. V. Moshchalkov, Phys. Rev. B 73, 014507 (2006)

85. C. C. de Souza Silva, J. Van de Vondel, M. Morelle, and V. V. Moshchalkov, Nature 440, 651 (2006) 